\title{
Emprendimiento cooperativo de trabajo: resultados, expectativas y desempeño empresarial. Un análisis en cooperativas valencianas de trabajo asociado
}

\author{
Manuel Monreal Garrido \\ Waldo E. Orellana Zambrano
}

RESUMEN: El contexto de las cooperativas de trabajadores mantiene importantes diferencias con el de las empresas tradicionales, y el estudio del emprendimiento difiere también de forma sustancial. El análisis de la creación y puesta en marcha de las cooperativas de trabajo asociado requiere enfoques diferenciados, ya que los modelos más convencionales de entrepreneurship no resultan adecuados. Tales modelos, de carácter multidimensional en su mayor parte, recogen factores que resultan determinantes para explicar el proceso emprendedor, aunque no es frecuente encontrar en esos modelos una relación causal de los factores con los resultados del emprendimiento. Desde el enfoque sociocultural y la orientación emprendedora, en este trabajo se analiza la influencia o relación de algunos factores presentes en el proceso de emprendimiento sobre dichos resultados, tanto los referidos al desempeño o la performance de la propia empresa, como a las expectativas o resultados personales esperados por parte de dichos emprendedores. Con una muestra de cooperativas de trabajo asociado creadas en la Comunidad Valenciana (España) en el periodo 2008-2014, y mediante una metodología cuantitativa, se realiza un análisis exploratorio para comprobar la relación o influencia de los factores estudiados sobre los resultados del emprendimiento.

PALABRAS CLAVE: Cooperativas, Trabajo asociado, Orientación emprendedora, Emprendimiento, Performance, Ecuaciones estructurales.

CLAVES ECONLIT: L26, M1, M20, M54.

Cómo citar este artículo / How to cite this article: MONREAL, M. \& ORELLANA, W. (2019): "Emprendimiento cooperativo de trabajo: resultados, expectativas y desempeño empresarial. Un análisis en cooperativas valencianas de trabajo asociado", CIRIEC-España, Revista de Economía Pública, Social y Cooperativa, 97, 5-47. DOI: $10.7203 /$ CIRIEC-E.97.12558.

Correspondencia: Manuel Monreal Garrido, IUDESCOOP, Universitat de València, manuel.monreal@uv.es; Waldo Ezequiel Orellana Zambrano, IUDESCOOP, Universitat de València, Waldo.orellana@uv.es. 


\section{EXPANDED ABSTRACT}

\section{Cooperative work venture: results, expectations and business performance. An analysis of Valencian worker cooperatives}

\section{Contextualisation and aims}

This research adopts the initial, general hypothesis that the entrepreneurial process of the worker cooperative, in its various stages of development and establishment, has some unique features in comparison to the traditional entrepreneurial model, which has an impact on the results of the cooperative and the entrepreneurs. This hypothesis is justified by the fact that there are significant organisational differences between the worker cooperative and the traditional capitalist company due to the majority of the cooperative's members acting as both owners and workers, thereby establishing business relationships between equals and circularity in principal-agent relationships. This has been shown by various studies.

These differences are characteristics inherent to the worker cooperative that determine a unique and distinctive organisational context that will affect the cooperative's management processes. Based on this organisational uniqueness, this study aims to contribute to the understanding of business behaviour by investigating the entrepreneurial process of the worker cooperative and its relationship to company performance and the expectations of the cooperative entrepreneurs. Bearing in mind that, for these people, the main aim of their entrepreneurial activity is achieving personal and professional development using a participative business approach.

In line with the above, from a multidimensional perspective, a model has been created that analyses this business behaviour, or entrepreneurial orientation, using a set of factors obtained from the most relevant theories within the sociocultural and institutional approach and that are present in the entrepreneurial process in the inception, creation and implementation phases of the cooperative.

In this manner, the impact of sociocultural factors on the results of the cooperative entrepreneurship can be studied, analysed through two components or constructs: a) material results arising from the cooperative's performance (and which fundamentally affect it), and b) the level of achievement or meeting of expectations, or personal results, which are much more important for the entrepreneurs.

The relationship between or influence of the sociocultural factors of entrepreneurship on the results or performance of worker cooperatives is studied based on thought within the research area known as entrepreneurial orientation, based on a multidimensional construct that includes the constant search 
for innovation, proactiveness and predisposition to take moderate risks (and to which some authors have subsequently added new dimensions to the initial construct).

\section{Methodology and research design}

The research used information provided by 103 worker cooperatives in the Valencian Community, selected from the 633 created in the period from 2008 to 2014. The information was obtained using questionnaires structured into various sections that were answered by the founders and managers of the cooperatives. The methodology used in the research is based on a model of structural equations, having used the PLS-SEM tool, which is suitable for studies with small sample sizes using an exploratory approach, as is the case here.

In accordance with various studies on entrepreneurial orientation and its relationship to business performance, the aim was to connect the sociocultural factors to some of the dimensions of the entrepreneurial orientation construct, and analyse its influence on the performance and results of cooperative entrepreneurship, presenting a specific model based on the multidimensional approach of entrepreneurial orientation. With this goal, an exploratory study using structural equations was chosen in order to explain the relationship between the factors that determine the entrepreneurial process and the results of entrepreneurship.

The selected sociocultural factors were: reasons for entrepreneurship, the entrepreneurs' prior experience, relationships with local agents, attractiveness of the sector and the influence of the contractual form of the cooperative, each one of them connected to a theory from the sociocultural approach. With regards to the results of the venture, these were presented as two concepts: the cooperative's performance and the meeting of the members' personal expectations.

The empirical study analyses the direct influence of sociocultural factors on both entrepreneurship result types, as well as the indirect or mediation relationship between the sociocultural factors and the personal results when the other result variable, performance of the company, acts as the mediator in this relationship.

In addition, the restraining effect of factors that are independent of each other was studied, in terms of their relationship to or influence on the entrepreneurial result variables, in accordance with other studies on entrepreneurial orientation that have carried out similar analysis (Green, Covin and Slevin, 2008; Stam and Elfring, 2008). The study was completed by verifying the effects that a group of demographic control variables may have on the model. 


\section{Results and practical conclusions}

The main aim of this study was to clarify the multidimensional nature of the entrepreneurial process in worker cooperatives, as well as to suggest alternative contingency models that, similarly to other studies on entrepreneurial orientation, may provide additional information on the entrepreneurial orientation-performance relationship. In summary, it is worth highlighting the following results regarding the sociocultural factors studied:

The reasons for entrepreneurship factor is the only one that directly or significantly influences the personal results, without any kind of impact or influence on the cooperative's performance, while the prior experience and attractiveness of the sector only affect the levels of performance in the company without any significant influence on the personal results. The other factors have a direct and significant influence both on the improvement of performance as well as the personal results.

With regards to possible mediating effects, the results of the analysis confirm that the business performance factor has a mediating effect on the indirect relationship between the independent factors, including the control variables and the personal results, this mediating relationship being significant, with the exception of the reasons for entrepreneurship factor.

Regarding the behaviour of the demographic control variables, the results show an intermediate or moderate predictive relevance for all of these considered as a whole with regards to the personal results variable, and a wide or strong predictive relevance with regard to the business performance variable.

In terms of the moderation and interaction between factors, the variables analysed as a whole verify that the entrepreneurs' prior experience of a working, professional or business nature moderates the influence that establishing relations with local agents has on business performance.

In terms of the conclusions, and considering that the worker cooperative does not fit exactly with the standards of entrepreneurship in traditional businesses and that its entrepreneurial process can be considered a new phenomenon within this research programme, this study has taken this restriction into account and contributed to the understanding of business behaviour, materialised through the entrepreneurial process of the worker cooperative, and its relationship to the businesses' performance and the expectations of the cooperative entrepreneurs.

The aim of this study was to build on prior research and theories in order to, firstly, clarify the multidimensional nature of the entrepreneurial process in worker cooperatives, and secondly to suggest alternative contingency models that, similarly to other studies on entrepreneurial orientation, may provide additional information on the entrepreneurial orientation-performance relationship. 
It is for this reason that, from this multidimensional perspective, a unique model is proposed that analyses this business behaviour, or entrepreneurial orientation, using a group of factors selected from the most relevant theories within the sociocultural and institutional approach, these being present in the business process. The research efforts aimed at understanding the dimensionality of the business process of worker cooperatives will contribute to a greater development of the theory in the field of entrepreneurship.

KEYWORDS: Cooperatives, Associated Work, Entrepreneurial Orientation Entrepreneurship, Performance, Structural Equations.

\section{Introducción 1}

La cooperativa de trabajadores (en adelante CTA), que en la bibliografía anglosajona es conocida como worker cooperative ${ }^{2}$, al igual que la empresa capitalista tradicional, se enmarca en el llamado modelo de producción en equipo (Alchian y Demsetz, 1972), si bien, y desde un punto de vista organizativo, mantiene importantes diferencias con respecto a esta última en cuanto al sistema decisor, al comportamiento previsto de sus miembros, sus incentivos y sus relaciones laborales.

Así, el sistema decisor está determinado por sus atributos contractuales, que muestra diferencias sustanciales con el contexto de la empresa capitalista tradicional donde están claramente separados propiedad y control (Fama y Jensen, 1983), mientras que en la cooperativa de trabajo no existe esa separación, y los trabajadores tienen idénticos derechos de propiedad sobre la empresa, consecuencia de las relaciones entre iguales (Orellana, 2002).

De igual forma, el comportamiento de sus miembros responderá preferentemente a los incentivos del tipo valores de conservación (March y Simon, 1958), más relacionados con los objetivos y el crecimiento de la empresa, dentro de un contexto de amplia responsabilidad y discrecionalidad en su actuación (Simon, 1947). Todo ello por la participación de los miembros en la dirección de la empresa, ya sea directa o a través de los órganos representativos.

1.- Este trabajo se enmarca en el Proyecto GV-2017-002 de la Consellería d'Educació, Investigació, Cultura i Esport .

2.- Y que en España su denominación legal es la de cooperativa de trabajo asociado, utilizándose también el acrónimo CTA en la literatura española para referirse a este tipo de organizaciones. 
En lo concerniente a las relaciones laborales-contractuales, en la CTA predominan las estructuras de gobierno del tipo equipo de relación (Williamson, 1985) ${ }^{3}$, con un predominio de trabajadores idiosincrásicos en un contexto laboral participativo y comunicativo, porque la experiencia y la información compartida, las relaciones de confianza y la convergencia entre los intereses particulares y los objetivos de la empresa cooperativa, añaden al trabajador características idiosincrásicas difíciles de encontrar en el mercado laboral.

Por tanto, el sistema decisor, el comportamiento de los miembros y las relaciones laborales en la CTA configuran un contexto organizativo singular y distintivo, que afectará a los procesos de dirección de la cooperativa ${ }^{4}$ por lo que cabe adoptar como hipótesis que el proceso de emprendimiento en sus distintas fases de desarrollo y consolidación presenta, asimismo, ciertas singularidades con relación al modelo tradicional de emprendimiento, con una incidencia especial en los resultados de las empresas y de los emprendedores.

El emprendimiento cooperativo de trabajo, aunque no aparece de forma explícita en los modelos más conocidos ${ }^{5}$ de emprendimiento 0 entrepreneurship, puede ser abordado perfectamente por el enfoque sociocultural e institucional, ya que éste es uno de los enfoques más relevantes en el ámbito del emprendimiento, y las teorías que recoge están también incluidas en el dominio del entrepreneurship.

Por otra parte, para estudiar la relación o influencia de los factores del emprendimiento sobre los resultados o el rendimiento de las CTA, se consideran en este trabajo algunas aportaciones de la corriente de investigación sobre la orientación emprendedora.

Tras plantear el marco conceptual y las condiciones particulares que se dan en el estudio del emprendimiento en este tipo de organizaciones, el presente trabajo pretende hacer una contribución estudiando cómo afectan algunos factores determinantes en la creación de CTA en los resultados del emprendimiento cooperativo, los cuales son analizados en dos componentes o constructos: por una parte aquellos resultados materiales derivados del desempeño o performance de la empresa, los cuales afectan fundamentalmente a ésta, y por otra, el nivel de logro o de cumplimiento de las expectativas, o resultados personales, más propios de, y que afectan en mayor medida, a los emprendedores.

3.- Williamson (1985) denomina estructuras de gobierno del trabajo a las distintas formas con las que cuenta la empresa para contratar y establecer los incentivos. En este marco, las estructuras denominadas equipo de relación son aquellas orientadas a gobernar el trabajo idiosincrásico cuyo output es el resultado del trabajo en equipo del trabajador y por ello resulta difícil determinar la productividad individual y por tanto los incentivos colectivos son los que deben prevalecer.

4.- La justificación de cómo los atributos institucionales generan un contexto singular exclusivo de las cooperativas de trabajadores, puede verse en Orellana (2002).

5.- Shapero (1975), Shapero y Sokol (1982), Gartner (1985), Hayton et al. (2002), Verheul et al. (2002). 
El estudio ha sido realizado con una muestra representativa de 103 CTA, de un total de 633 cooperativas creadas en la Comunidad Valenciana (España) durante los años 2008 a 2014, un periodo marcado por el inicio de la reciente crisis económica, los años más difíciles de ésta, y el comienzo de la recuperación.

Sin que dicha crisis económica haya sido el motivo principal de este trabajo, se ponen en valor ciertas capacidades desarrolladas por los emprendedores cooperativos, y las características idiosincrásicas de éstos, en su afán de sobrevivir y hacer frente a la crisis.

Se opta por realizar un estudio exploratorio mediante ecuaciones estructurales, al no existir muchos trabajos cuantitativos en la investigación en emprendimiento cooperativo de trabajo asociado, y aún menos que expliquen la relación de factores determinantes del proceso emprendedor con los resultados del emprendimiento. En este trabajo se analizan dichas relaciones, directas, indirectas o de mediación, e incluso efectos de moderación entre factores, siendo también de aplicación en este trabajo, como variables de control, un conjunto de variables demográficas.

El presente artículo se estructura de la siguiente manera: además de esta introducción, el segundo apartado trata de establecer el marco teórico para el emprendimiento en este tipo de organizaciones, exponiendo los factores socioculturales a estudiar, presentes en el emprendimiento cooperativo, haciendo de paso una breve revisión del concepto de orientación emprendedora, con el objeto de establecer las relaciones entre los factores del emprendimiento y el rendimiento, desempeño 0 performance de las CTA, para terminar exponiendo un modelo conceptual y las hipótesis teóricas que se presentan. En el tercer apartado se desarrolla la metodología y se describen las variables y constructos presentes en el modelo estructural, los factores independientes, factores dependientes y variables de control, mientras que el cuarto apartado presenta el análisis y discusión de los resultados. Finalmente, en el quinto apartado se exponen unas breves conclusiones.

\section{Emprendimiento en las cooperativas de trabajo asociado. Marco conceptual}

\subsection{Factores socioculturales en el proceso emprendedor de las CTA}

Las teorías recogidas en el enfoque sociocultural sostienen que la constitución de nuevas empresas, en este caso cooperativas de trabajadores, tiene su origen en factores externos, siendo los factores de carácter sociocultural y el marco institucional los que pueden resultar favorables para la creación de nuevas empresas o la decisión de convertirse en empresario, y las percepciones sobre el entorno suponen un componente crucial en la decisión de una persona a la hora de crear su propia empresa. 
Las teorías más representativas de este enfoque son, además de la teoría institucional, la teoría de la marginación, la teoría del rol, la teoría de redes, y la teoría de la organización incubadora 6 .

La Teoría institucional proporciona un marco conceptual robusto y apropiado para el estudio de la influencia de los factores ambientales en la función empresarial y en la creación de empresas. Distingue entre factores formales (normas políticas, legales, económicas y contratos), e informales.

Los factores formales definen el marco de actuación, las pautas de comportamiento y el grado de discrecionalidad en las actuaciones ${ }^{7}$, mientras que los factores informales actúan como telón de fondo, ya que gran parte de las actuaciones de los agentes económicos están guiadas por factores informales, estando relacionados con aspectos más culturales y sociales.

Por otro lado, según la Teoría de la marginación, la creación de una empresa comienza debido a una discontinuidad o desplazamiento negativo (Shapero, 1975), o un suceso crítico, generalmente negativo, que precipita los acontecimientos en lo que se denomina deterioro del rol (Collins et al., 1964), o un suceso disparador o trigger event (Shapero, 1982), siendo las personas más propensas y proclives a convertirse en empresarios, según esta teoría, aquellas que están inadaptadas o marginadas. En este sentido, resultados de investigaciones como las de Evans y Leighton (1989), y de Bathes (1997), han venido a confirmar que la decisión de crear una empresa está motivada por ese suceso negativo, que generalmente desencadena y/o precipita el proceso de creación de una empresa. Según esta teoría, y como afirman Brunet y Alarcón (2004), para convertirse en empresario son necesarios dos requisitos: a) un periodo de maduración de la idea desde un tiempo anterior; y b) un evento disparador, desencadenante de la creación, que no responda necesariamente a una lógica de aprovechar una oportunidad o de obtener un beneficio, sino más bien estar provocado por un suceso crítico de carácter negativo, como una situación prolongada de desempleo, un despido, una falta de seguridad en el empleo, o simplemente un desencuentro con las ideas y objetivos de la empresa que actualmente le proporciona trabajo al individuo, lo que le hace a éste abandonar la misma (Tervo, 2006).

En cuanto a la teoría de la organización incubadora, esta teoría está basada en que muchas ideas que han llevado a la creación de una nueva empresa están relacionadas directamente con la ocupación, experiencia y conocimientos adquiridos previamente en la empresa donde el socio fundador de la cooperativa ha trabajo con anterioridad. Una vertiente de esta teoría analiza la necesidad de crear y desarrollar empresas, las denominadas spin-off, lo que ha animado a Administraciones Públicas, Universidades, Cámaras de comercio, empresas privadas e incluso a algunas organizaciones no gubernamentales, a establecer y desarrollar programas de incubadoras (OCDE, 1999).

6.- Entre las aportaciones principales en el ámbito de las teorías del rol, de redes, y de la organización incubadora cabe subrayar los trabajos de Cooper (1985), y de Cooper y Dunkelberg (1987).

7.- Williamson (1985) en el campo de la economía de las organizaciones y North (1990) en el campo de la teoría económica son los autores de referencia en el estudio de los aspectos institucionales como condicionantes de la economía. 
No obstante, la mayor parte de las investigaciones sobre la organización incubadora analiza la relación o conexión entre las empresas en las que ha estado trabajando el emprendedor con anterioridad y la nueva empresa creada por éste, considerando que las primeras han servido de incubadoras para el nuevo proyecto empresarial, donde el emprendedor llega a descubrir y a materializar una idea de negocio (Westhead y Wright, 1998).

La teoría del rol, por su parte, viene a explicar, entre otros aspectos, algunas de las razones por las cuales en determinadas áreas geográficas se crean más empresas que en otras (explicando por qué en las regiones industriales donde existe una "tradición empresarial", o en todo caso un tejido industrial, se crean nuevas empresas). La existencia de modelos emprendedores a seguir en un determinado entorno geográfico produce un efecto de arrastre que estimula o induce la aparición de nuevos empresarios (Nueno, 1996). Es decir, es una situación en la que el futuro empresario advierte que otros individuos en circunstancias similares a las de él han logrado crear su propia empresa, y éste decide emprender el mismo cometido (Veciana, 1996).

En lo que respecta a la teoría de redes, intenta explicar el papel de las redes sociales en el proceso de creación de la empresa, poniendo el énfasis en que las redes son vitales para percibir oportunidades y obtener recursos, y constituyen elementos críticos de las organizaciones emergentes. Esta teoría adquirió importancia en la década de 1980, dando lugar a una corriente de investigación alrededor del impacto de la red (network) en el proceso de creación de empresas, aunque a pesar de la extensa literatura que viene a relacionar las redes sociales con la creación de empresas, en realidad son pocas las investigaciones que han llegado a explorar la existencia y estructura de redes formales de empresas y sus verdaderas implicaciones en el proceso para el desarrollo emprendedor (Minniti, 2005).

La teoría de redes plantea que el conjunto de relaciones específicas entre varios grupos 0 actores proporciona múltiples interconexiones y reacciones en cadena, cuyo resultado consiste en hacer circular la información y las ideas, y facilitar al emprendedor la creación de la empresa (Alonso y Galve, 2008). Existe una gran variedad de tipos de redes según la consideración de distintos autores. Birley (1985), distingue entre redes formales (bancos, profesionales, cámaras de comercio) e informales (familias, amigos, compañeros de trabajo), afirmando que los nuevos empresarios se sirven más de estas últimas que de las redes formales.

El cuadro 1 recoge los factores socioculturales del proceso de emprendimiento en las CTA que van a ser objeto de estudio de este trabajo, relacionados cada uno de ellos con alguna de las teorías socioculturales anteriores, y cuyos constructos serán desarrollados en el tercer apartado. A continuación, se realiza una breve descripción de cada factor, mientras que en la siguiente sección se describe brevemente el concepto de orientación emprendedora y su influencia en el desempeño, en relación a dichos factores. 


\section{Cuadro 1. Factores determinantes del proceso emprendedor, y teorías relacionadas}

\begin{tabular}{|c|c|}
\hline Factor determinante del proceso de emprendimiento & Teoría relacionada \\
\hline Causas para el emprendimiento & $\begin{array}{l}\text { Teoría de la marginación } \\
\text { Teoría de la organización incubadora }\end{array}$ \\
\hline Experiencia previa de los emprendedores & Teoría de la organización incubadora \\
\hline Relaciones con los agentes del entorno & Teoría de redes \\
\hline Atractivo del sector & Teoría del rol \\
\hline Forma contractual & Teoría institucional \\
\hline $\begin{array}{l}\text { Nivel formativo de los emprendedores } \\
\text { Edad de los emprendedores } \\
\text { Género de los emprendedores }\end{array}$ & Variables demográficas de control \\
\hline
\end{tabular}

FUENTE: Elaboración propia.

\subsubsection{Causas para el emprendimiento}

Este factor recoge las posibles causas u orígenes para el iniciar el emprendimiento, a modo de factor push, que obliga a una persona a emprender, como así lo han puesto de manifiesto teorías como la teoría de la marginación y la teoría de la organización incubadora (Orellana y Martínez de Lejarza, 2013). Esto es habitualmente uno de los argumentos principales para emprender en el ámbito cooperativo, aunque no sería el único factor, al existir en la iniciativa de creación de CTA otros factores del entorno tipo pull o de oportunidad. Las causas o situaciones más comunes que empujan a emprender bajo una situación de cierta necesidad serían el desempleo, tanto friccional como de larga duración, incluso en una situación de exclusión social. Otra causa para crear una CTA sería una situación de insatisfacción en la condición de trabajador por cuenta ajena o de trabajador autónomo. Otra causa conocida es el acontecimiento de una crisis en la empresa en la que ha estado trabajando y los trabajadores asumen el control de la misma, sea ésta una empresa capitalista tradicional, u otra cooperativa. También suelen emprender personas que, tras haber finalizado sus estudios deciden crear una nueva CTA, ante la incertidumbre de encontrar un empleo que satisfaga sus aspiraciones. Esa opción también puede considerarse un motivo push, que completaría la lista sobre orígenes o causas para el emprendimiento.

\subsubsection{Experiencia previa de los emprendedores}

Este factor, desde la perspectiva de la organización incubadora, recoge la relación y la experiencia previa de los emprendedores en la empresa, y los conocimientos adquiridos supuestamente en empleos anteriores, haciendo referencia de manera conjunta a la experiencia laboral por cuenta ajena y a la experiencia profesional, incluso directiva o de gestión, que ha adquirido el individuo antes de tomar la decisión de emprender. 


\subsubsection{Relaciones con los agentes del entorno}

Desde la perspectiva de la teoría de redes, se plantea este factor, que recoge relaciones que la empresa ha conseguido establecer de manera eficaz con dichos agentes, como forma de atesorar capacidades, intangibles en forma de capital relacional, y comprobar su impacto sobre los factores de resultado.

\subsubsection{Atractivo del sector}

Como se ha señalado anteriormente, la teoría del rol viene a explicar las razones por las que en unas áreas geográficas se crean más empresas que en otras, dando razones de por qué en las regiones industriales donde existe una "tradición empresarial", o en todo caso un tejido industrial, se crean nuevas empresas, y cómo la percepción y la existencia de un cierto atractivo del sector y de modelos emprendedores a seguir en un determinado entorno geográfico produce un efecto de arrastre en la que el futuro empresario advierte que otros individuos, en circunstancias similares a las de él han logrado crear su propia empresa.

\subsubsection{Forma contractual}

Por último, se plantea este factor desde la teoría institucional, que proporciona un marco conceptual consistente y apropiado para estudiar la influencia de factores ambientales en la función empresarial y en el emprendimiento. Cuando se trata de cooperativas de gran tamaño, en las que existe un número importante de interacciones entre diferentes órganos de gobierno, gestión y control de la cooperativa, el análisis de estos factores, tanto organizativos como contractuales, tendrá una mayor relevancia.

\subsection{Factores socioculturales, orientación emprendedora y resultados en las CTA}

Para el análisis de la influencia de los factores socioculturales sobre el desempeño y el rendimiento de las CTA, se va a considerar la corriente de investigación conocida como orientación emprendedora (entrepreneurial orientation), teniendo en cuenta algunas de sus aportaciones más relevantes para nuestro análisis.

El concepto de orientación emprendedora es considerado ampliamente como una piedra angular de la literatura sobre emprendimiento a nivel de empresa, y se refiere a una postura organizacional estratégica que captura los procesos, prácticas y actividades específicas que permite crear valor en la empresa mediante actividades emprendedoras (Lumpkin y Dess, 1996; Wales, Gupta y Mousa, 2013).

En los últimos años ha sido objeto de estudio en la literatura de empresa, y ha recibido una importante atención, tanto conceptual como empírica, siendo ya una de las áreas de investigación donde más se está desarrollando un cuerpo acumulativo de conocimientos, centrándose la mayoría de los 
trabajos en analizar el efecto de la orientación emprendedora en el desempeño empresarial (Hernández Perlines, 2014), es decir, considerándola como una variable independiente, si bien, en otro conjunto de trabajos sus autores se han centrado en los factores internos y externos que pueden influir en la orientación emprendedora de la empresa. Es decir, considerada en este caso como una variable dependiente (Arzubiaga, Iturralde y Maseda, 2012).

De cualquier forma, a la hora de definir la orientación emprendedora, la perspectiva de Shumpeter (1934) ha sido un punto de partida y de referencia en las propuestas para su conceptualización. Miller (1983), que fue el primer autor en hablar de orientación emprendedora, la definió como un constructo multidimensional en el que se integran la búsqueda constante de innovación, proactividad y predisposición a aceptar riesgos moderados, dimensiones éstas que tenían que covariar positivamente entre ellas (Covin y Wales, 2012).

Más tarde, Covin y Slevin (1989) completaron la definición de Miller, afirmando que "la orientación emprendedora de una empresa se demuestra por el grado en que la alta dirección está dispuesta a asumir los riesgos relacionados con la empresa (dimensión de asunción de riesgos), para favorecer el cambio y la innovación con el fin de obtener una ventaja competitiva para su empresa (dimensión de innovación), y competir agresivamente con otras firmas (dimensión de proactividad) ".

Posteriormente Lumpkin y Dess $(1996,2001)$ extendieron el dominio de este constructo al añadir dos dimensiones adicionales: autonomía y agresividad competitiva, realizando dichos autores una importante clarificación del constructo multidimensional, en cuanto a que las dimensiones que lo componen no necesitan covariar entre sí para calificar a una empresa como emprendedora. Es decir, una empresa puede llegar a obtener altas puntuaciones en alguna dimensión, y bajas en otra, dependiendo de ciertas variables y condiciones contingentes, fundamentalmente del entorno (Lawrence y Lorsch, 1967), sin poner en duda por ello la condición emprendedora de la empresa.

Dicho criterio fue adoptado por numerosos autores en sus siguientes investigaciones, y a partir de ese momento surgió un importante número de trabajos, conceptuales y empíricos, con las tres dimensiones iniciales, o las cinco, siendo los primeros los más numerosos, en los que se confirmó la existencia de relaciones positivas entre la orientación emprendedora y el resultado empresarial (Rauch et al., 2009). Sin embargo, otros trabajos no han podido probar esta relación (Smart y Conant, 1994; George, Wood y Khan, 2001), o incluso otros en los que, en determinadas circunstancias, existen incluso relaciones negativas (Hart, 1992).

Diferentes estudios han analizado los factores relacionados con actividades o procesos y su influencia en el desempeño o rendimiento de las empresas, equiparándolos a alguna de las dimensiones de la orientación emprendedora, como la actividad exportadora de las cooperativas agroalimentarias (Hernández Perlines, 2014), el papel de las redes personales del empresario como proveedoras de recursos valiosos (Ripollés y Blesa, 2006), o las capacidades de aprendizaje organizativo en el desempeño innovador (Fernández-Mesa, Alegre y Chiva, 2012), entre otros. 
Otros estudios han buscado nuevos factores que expliquen esta relación, proponiendo algunos de ellos variables moderadoras, como son por ejemplo la estructura empresarial (Green, Covin y Slevin, 2008), o el capital social (Stam y Elfring, 2008). Mientras que otros proponen variables mediadoras como por ejemplo la orientación de aprendizaje (Wang, 2008).

Para concluir esta sección, cabe destacar que este trabajo, en la línea de otros sobre la orientación emprendedora y su relación con el desempeño empresarial, pretende equiparar los factores socioculturales estudiados con algunas de las dimensiones del constructo de la orientación emprendedora, y analizar su influencia en el desempeño y los resultados del emprendimiento cooperativo, presentando aquí un modelo particular basado en el planteamiento multidimensional de la orientación emprendedora.

Así, por ejemplo, el atractivo del sector tendría una cierta correspondencia con la innovación, la proactividad y la agresividad competitiva. Las relaciones con agentes del entorno y la experiencia de los emprendedores tendrían muchos aspectos en común con la innovación. La forma contractual tendría una cierta correspondencia con la autonomía o la predisposición a asumir riesgos, y finalmente el factor causas para el emprendimiento tendría también alguna relación con más de una dimensión, como la autonomía, la proactividad, o la asunción de riesgos.

\subsection{Modelo de investigación e hipótesis teóricas}

Los factores determinantes del proceso de emprendimiento de las CTA recogidos en el cuadro 1 , relacionados con las teorías socioculturales expuestas, y equiparados a alguna de las anteriores dimensiones de la orientación emprendedora, constituyen las variables independientes o exógenas en el modelo de investigación propuesto en esta sección, siendo representados como constructos o variables latentes en el modelo estructural posterior. Dicho modelo es presentado aquí a nivel conceptual, al igual que las hipótesis teóricas, el cual será desarrollado con un mayor nivel de especificación en el siguiente apartado, ya como modelo estructural. Los constructos y sus indicadores, que suponen su escala de medida, serán también debidamente expuestos y justificados.

Un aspecto crucial a destacar es la vinculación de mayor permanencia de los socios a la cooperativa, su calidad de socios y los conocimientos que tienen de la empresa, lo que hace que su trabajo adquiera un carácter idiosincrásico, al ser difícilmente transferibles a otra organización los valores y el sentimiento de clan desarrollados en ella (Ouchi, 1980), teniendo sus incentivos un importante componente cualitativo, basados más en valores de conservación y supervivencia de la empresa.

En este contexto, el presente trabajo realiza una aportación consistente en desdoblar y representar los resultados del emprendimiento de las CTA en dos constructos. En primer lugar, el resultado propio de la empresa, que es consecuencia del desempeño colectivo, y equivale al concepto tradicional de performance de la empresa y, en segundo lugar, los resultados personales, que se refieren 
al grado de cumplimiento de las aspiraciones y expectativas individuales de los promotores, más en la línea de los anteriores aspectos cualitativos.

El trabajo empírico posterior analiza la influencia directa de los factores socioculturales sobre ambos tipos de resultado del emprendimiento, así como la relación indirecta, o de mediación, entre los factores socioculturales y los resultados personales cuando la otra variable del resultado, desempeño o performance de la empresa actúa como mediadora en dicha relación.

De forma adicional, se estudia el efecto de moderación de factores independientes entre sí, en su relación o influencia sobre las variables del resultado emprendedor 8 , en la misma línea de otros trabajos sobre orientación emprendedora que han realizado análisis similares (Green, Covin y Slevin, 2008; Stam y Elfring, 2008), completándose el trabajo con la comprobación de los efectos que pueden tener en el modelo un conjunto de variables demográficas de control.

La figura 1 representa, de forma conceptual y resumida, el modelo de investigación para un solo factor, en su relación directa con ambos factores del resultado, y en su relación indirecta, cuando la variable desempeño media en la relación entre el factor y la variable resultados personales. El modelo estructural recoge todos los factores simultáneamente.

\section{Figura 1. Modelo conceptual de investigación e hipótesis teóricas para un solo factor}

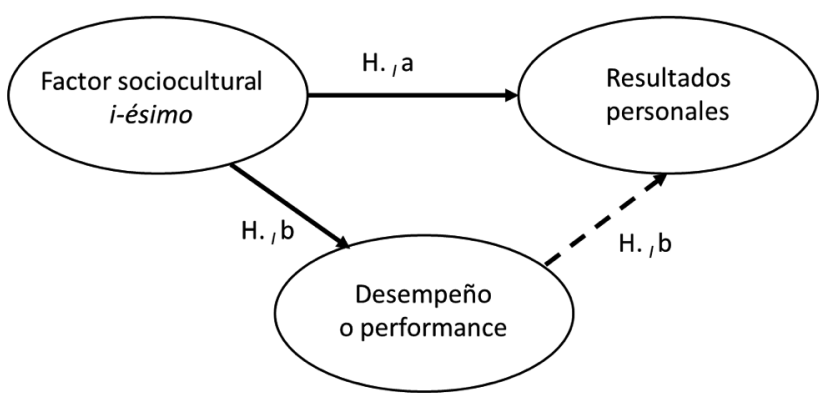

FUENTE: Elaboración propia. 
De forma genérica, las hipótesis teóricas a establecer para cada factor sociocultural y para cada variable de control, a contrastar en el trabajo empírico, serían las siguientes:

H. $;$ a: El factor $i$ tiene una influencia directa sobre los resultados personales de los emprendedores

H. $;$ b: El factor $i$ tiene una influencia directa sobre los el desempeño o performance de la CTA, e indirecta sobre los resultados personales de los emprendedores, a través de la mediación del desempeño

Cabría citar una serie de referencias teóricas, tratando de justificar, para cada uno de los factores socioculturales y variables de control, las relaciones individuales establecidas en las distintas hipótesis, de las cuales, algunas de ellas resultan muy obvias. Dichas referencias pueden encontrarse en la abundante literatura que existe ya sobre orientación emprendedora, Por lo que, más que justificar a nivel teórico las relaciones o influencia a nivel individual de dichos factores, lo que resulta más interesante es analizar de manera conjunta, y desde una perspectiva multidimensional, la posible influencia de los factores socioculturales, de forma combinada, sobre los resultados del emprendimiento.

\section{Metodología y desarrollo de la investigación}

3.1. Ficha técnica de la investigación. Población y muestra

\section{Cuadro 2. Ficha técnica de investigación}

\begin{tabular}{|c|c|}
\hline Descripción del Universo & 633 CTA creadas en el periodo 2008-2014 \\
\hline Metodología de selección de la muestra & Muestreo aleatorio simple \\
\hline Tamaño de la muestra & 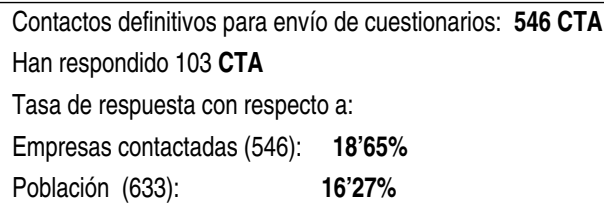 \\
\hline $\begin{array}{l}\text { Margen de confianza y margen de error de los resultados } \\
\text { presentados }\end{array}$ & $\begin{array}{l}\text { Errores: } \quad{ }^{* *} p<0,001 \quad{ }^{* *} p<0,01 \quad{ }^{*} p<0,05 \\
\text { Niveles de confianza: }\end{array}$ \\
\hline Técnicas aplicadas para realizar el trabajo de campo & Cuantitativa: cuestionarios cumplimentados por las CTA \\
\hline Período de recogida de la información & Diciembre de 2014 a junio de 2015 \\
\hline
\end{tabular}

FUENTE: Elaboración propia. 
En la figura 2 y en el cuadro 3 se recogen la distribución sectorial por actividades, y sus correspondientes epígrafes del CNAE-2009 de las CTA creadas, siendo en éstas 2.147 el número total de socios fundadores, con una media de 3,4 socios. La mitad de las CTA que se han creado en dicho periodo corresponden al sector terciario (51'97\%), mientras que industria y la construcción han tenido una creación de empresas similar, en torno al 12'5\%. La actividad de comercio ha supuesto un 18'64\%, y sólo un 4'42\% las actividades relacionadas con la agricultura, que tradicionalmente se concentran en dos provincias, Castellón y Valencia.

En cuanto a una contextualización de los emprendedores cooperativos referida a media de edad, distribución por género, y nivel educativo de los mismos, esta información se detalla en la sección dedicada a la exposición de variables demográficas de control.

\section{Figura 2. CTA creadas entre 2008 y 2014. Distribución por sectores de actividad}

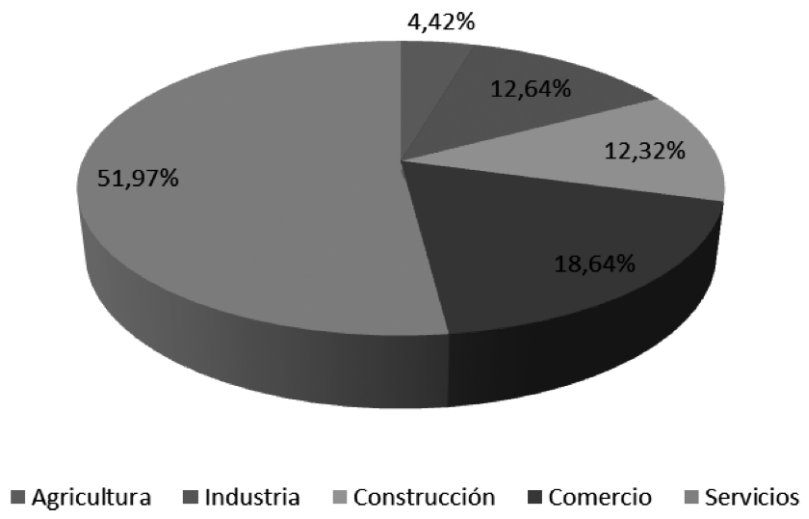

FUENTE: Elaboración propia a partir de datos de FEVECTA.

El cuestionario de investigación se ha estructurado en diferentes secciones para recoger los apartados y bloques que contienen las variables manifiestas (indicadores) para medir los diferentes constructos, generalmente mediante escalas de Likert (1 ... 5), o bien dicotómicas en algunos casos, además de los bloques para datos identificativos y de segmentación, siendo entrevistados los socios fundadores, en la mayor parte de los casos aquellos quienes ostentaban el cargo de gerente, y en otros casos el presidente del consejo rector, no existiendo un criterio uniforme a priori en cuanto a la elección del entrevistado, sino que esto dependía de la disponibilidad de las personas para responder al cuestionario. 


\section{Cuadro 3. CTA creadas en la Comunidad Valenciana entre 2008 y 2014 (CNAE-2009)}

\begin{tabular}{|c|l|c|}
\hline Grupo & & Número \\
CNAE & TITULO & de CTA \\
\hline A & Agricultura, ganadería, silvicultura y pesca & 28 \\
C & Industria manufacturera & 80 \\
D & Suministro de energía eléctrica, gas, vapor y aire acondicionado & 3 \\
E & Suministro de agua, actividades de saneamiento, gestión de residuos y descontaminación & 3 \\
F & Construcción & 82 \\
G & Comercio al por mayor y al por menor; reparación de vehículos de motor y motocicletas & 123 \\
H & Transporte y almacenamiento & 23 \\
I & Hostelería & 56 \\
J & Información y comunicaciones & 34 \\
K & Actividades financieras y de seguros & 6 \\
L & Actividades inmobiliarias & 15 \\
M & Actividades profesionales, científicas y técnicas & 60 \\
N & Actividades administrativas y servicios auxiliares & 32 \\
O & Administración Pública y defensa; Seguridad Social obligatoria & 2 \\
P & Educación & 32 \\
Q & Actividades sanitarias y de servicios sociales & 21 \\
R & Actividades artísticas, recreativas y de entretenimiento & 21 \\
S & Otros servicios & 10 \\
T & Actividades de los hogares como empleadores de personal doméstico; & 2 \\
& actividades de los hogares como productores de bienes y servicios para uso propio & \\
\hline & TOTALES & 2 \\
\hline
\end{tabular}

FUENTE: Elaboración propia a partir de datos de FEVECTA.

3.2. Factores socioculturales en el proceso emprendedor de la CTA. Constructos, variables, indicadores y escalas

La metodología empleada en este trabajo está basada en un modelo de ecuaciones estructurales, que permite examinar una serie de relaciones de dependencia de forma simultánea. Dichos modelos están incluidos en el denominado análisis multivariante de segunda generación (Fornell, 1982; Hair et al, 2019), ya que dichos modelos suponen una metodología que utiliza e incluye tanto variables empíricas como constructos o variables abstractas, es decir, vincula datos y teoría, enfatizando aspectos acumulativos del desarrollo teórico, donde el conocimiento a priori ${ }^{9}$ es incorporado dentro del análisis empírico.

9.- Dicho conocimiento a priori puede originarse a partir de la revisión de la teoría, de descubrimientos empíricos previos, del proceso de reflexión del investigador o del propio diseño de la investigación. 
Dentro de la metodología de ecuaciones estructurales, se ha utilizado la herramienta PLS-SEM, adecuada para estudios con muestras reducidas, dentro de un planteamiento exploratorio, como es nuestro caso.

A diferencia de los modelos de estructuras de covarianzas (CEM), basados en el criterio de máxima verosimilitud, el algoritmo del PLS-SEM utiliza el criterio de mínimos cuadrados ordinarios (OLS), y su fundamento son las regresiones, siendo una ventaja adicional la inclusión de constructos formativos, junto a otros constructos reflectivos, dentro de un mismo modelo estructural, a diferencia de los modelos CEM, que sólo permiten utilizar factores reflectivos.

A excepción del factor causas para el emprendimiento, definido como un constructo formativo, el resto de factores independientes, variables de control y factores endógenos del resultado del emprendimiento, se definen como constructos reflectivos, por lo que deberán comprobarse en ellos las propiedades psicométricas en la evaluación del instrumento de medida y el modelo estructural, en cuanto a la fiabilidad, simple y compuesta, y a su validez, convergente y discriminante.

A continuación, se exponen de forma sucinta las variables manifiestas, o indicadores, que conforman las escalas de medida de los diferentes constructos independientes, y con un mayor nivel de detalle, se describen los constructos e indicadores de las variables demográficas de control y las variables dependientes del resultado del emprendimiento. A todos ellos se les asigna un acrónimo para identificarlos en el modelo estructural.

\subsubsection{Causas para el emprendimiento (CAUSEMP)}

Se plantean los siguientes indicadores de este constructo, los cuales conforman su escala de medida:

- X11: Personas en su primer empleo tras finalizar estudios

- X12: Situación de desempleo friccional, o de actividad como autónomo

- X13: Situación de desempleo de larga duración y/o exclusión social

- X14: Crisis en anterior empresa capitalista

- X15: Crisis en anterior cooperativa

Al tratarse de un constructo formativo, todas las causas para el emprendimiento han de ser significativos en su relación con el constructo, y no han de estar correlacionadas entre sí. De cada CTA encuestada se ha obtenido la distribución, en porcentaje, de socios fundadores de todos y cada uno de los orígenes o causas para el emprendimiento que contempla la escala de medida.

\subsubsection{Experiencia previa de los emprendedores (EXPREVI)}

Los siguientes indicadores evalúan el grado de experiencia en cada caso, mediante una escala de Likert 1-5 (valor 1: sin experiencia, valor 5: experiencia máxima):

- X21: Experiencia de tipo general. 
- X22: Experiencia técnica cualificada.

- X23: Experiencia directiva y de gestión.

\subsubsection{Relaciones con los agentes del entorno (RELAGEN)}

Cada indicador es medido mediante una escala de Likert 1-5, valorando entre 1: nula relación, y 5: estrecha relación, con dichos agentes. Las relaciones consideradas aquí se refieren las siguientes:

- X31: Proveedores (relaciones de carácter estable y a largo plazo)

- X32: Clientes (como clientela fija o fidelizada, al margen de cifras de ventas)

- X33: Empresas de diferentes sectores

- X34: Otras cooperativas

- X35: Asesores y empresas de consultoría

- X36: Asociaciones profesionales y empresariales

- X37: Entidades Públicas en general

\subsubsection{Atractivo del sector (ATRACTIV)}

Un estudio de mercado pormenorizado sería lo ideal para medir el posible atractivo de un sector, pero esto se escapa a las posibilidades de la mayor parte de estos pequeños emprendedores, planteándose un conjunto de preguntas, en forma de indicadores o variables manifiestas, para que estos puedan hacer su propia valoración del atractivo del sector, y que corresponden a las siguientes:

- X41: El sector donde la empresa opera tiene un gran atractivo

- X42: El sector donde la empresa opera depara muchas oportunidades

- X43: Ha resultado fácil establecerse y desarrollar la actividad en este sector

- X44: Se consiguen mejores resultados que los competidores

- X45: Resulta fácil captar y fidelizar a nuevos clientes

- X46: Capacidad negociadora con los clientes, en general

Cada indicador es medido con una escala de Likert 1-5, representando 1: un valor nulo o mínimo de atractivo, y 5: un valor máximo, y al tratarse de un constructo reflectivo, todos, o algunos de los indicadores, deben ser significativos con respecto al constructo, y ser, además, coherentes entre sí.

\subsubsection{Forma contractual (FCONTRAC)}

Sea el tamaño de la CTA que sea, con este factor latente se trata de analizar el efecto en la cooperativa de las relaciones de agencia circular, en el sentido de Jensen y Meckling (1976) y Fama y Jensen (1983), característica de esta forma organizativa, debido a la doble condición de propietarios $y$ trabajadores de sus socios, asumiendo en unos casos la figura del principal y en otros casos la del agente en la relación contractual. 
Para ello, se establece un conjunto de supuestos que reflejan distintas situaciones en las que se ejerce este tipo de relaciones de agencia, equivaliendo a los indicadores que miden este constructo:

- X51: Existe un fuerte control de la Junta Rectora o la Asamblea hacia la figura del gerente

- X52: Existe una importante contestación diaria de los socios a las decisiones operativas del gerente

- X53: Hay un alto grado de parentesco o de amistad previa entre los socios

- X54: Los miembros de la Junta rectora toman decisiones sesgadas, favoreciendo sus intereses personales

- X55: Han existido conflictos por decisiones polémicas del gerente o de la Junta Rectora

- X56: Suele haber consenso entre los órganos de gobierno, como norma general

\subsubsection{Variables demográficas de control}

Considerando el enfoque exploratorio de este trabajo, el propósito de utilizar variables de control, en este caso las variables demográficas género, edad y nivel formativo, es poder comprobar el efecto que van a tener éstas en el modelo planteado.

Existen evidencias sobre el efecto de esas mismas variables demográficas, que resultan determinantes para explicar la actuación de los emprendedores (Evans y Leighton, 1989; Verheul, 2005), aunque en ocasiones dichas evidencias han tenido un peso menor.

En ese sentido, Reynolds et al. (1995) señalan que la existencia de un importante porcentaje de población, entre 25 y 45 años, un rango de edad en el que las personas suelen estar buscando oportunidades para desarrollar su carrera profesional, y que no siempre encuentran, puede llevar a un mayor potencial emprendedor. La media de edad en la investigación de este trabajo es de 32,6 años, lo que viene a confirmar que los emprendedores cooperativos comienzan tardíamente su andadura emprendedora.

Para medir este constructo con un único indicador (X71: Edad de los emprendedores), se ha utilizado una escala de Likert 1-4, para representar la media de edad de los socios en el momento de la creación de la empresa, con los siguientes intervalos para la media de edad de los socios fundadores en el momento de la creación de la CTA (y sus valores de la escala de Likert):

- 1: Menor de 25 años

- 2: De 25 a 39 años

- 3: De 40 a 54 años

- 4: 55 años o más

En cuanto al género, y referido a las diferencias en estilos de liderazgo y capacidades de emprendimiento atribuidas a las diferencias de género, no existe un consenso en las investigaciones, dándose el caso que trabajos como los de Vecchio (2003) y Eagly y Carli (2003), que siendo de los más citados en la literatura especializada, obtienen resultados contrapuestos. 
En efecto, Vecchio (2003) a partir de una revisión de los resultados obtenidos en investigaciones sobre liderazgo y género, concluye que no existen evidencias que indiquen diferencias significativas entre hombres y mujeres, ni de que se haya podido probar la existencia de una clara superioridad por parte de uno de los sexos.

Por lo demás, en la presente investigación la distribución por géneros obtenida en el total de la muestra ha supuesto un 62 ' $8 \%$ de hombres y un $37 ' 2 \%$ de mujeres.

La medición de este factor de un solo indicador (X81: Género) se ha realizado con una distribución porcentual de hombres y mujeres que fundaron la cooperativa.

Finalmente, en cuanto al nivel formativo, la proporción de trabajadores con un nivel de educación superior entre la población (Armington y Acs, 2002), o de estudiantes e investigadores universitarios (Bosma et al., 2008; Reynolds et al., 1995), debería provocar una mayor tasa de creación de empresas. Se utiliza una escala de Likert 1-4 para medir el único indicador de este factor (X61: Nivel formativo de los emprendedores), que abarca desde los emprendedores sin estudios, hasta aquellos que han alcanzado un nivel universitario.

Los resultados globales en la investigación en cuanto a nivel formativo para cada una de las categorías, han sido los siguientes:

- 1: Sin estudios: $0^{\prime} 30 \%$

- 2: Estudios básicos de secundaria y primaria: $21{ }^{\prime 2} \%$

- 3: Bachiller y Formación profesional: $427 \%$

- 4: Estudios universitarios: 35 '8\%

Por último, cabe señalar que una variable tradicional para actuar como variable de control, como es el tamaño de la empresa, finalmente no se ha incluido en este trabajo dado el escaso tamaño de la práctica totalidad de empresas incluidas en la muestra, y por tanto el bajo grado de significatividad e incidencia que tendría esta variable en la varianza total de las variables del resultado emprendedor.

\subsection{Variables del resultado del emprendimiento en las CTA Constructos, indica- dores y escalas}

\subsubsection{Desempeño o performance empresarial (DESEMP)}

Una importante cuestión es la manera de medir la performance o el desempeño de la empresa que se adapte a las especifidades de las cooperativas de trabajo. March y Yagüe (2009), considerando los conceptos de efecto empresa y efecto sector, en línea con los trabajos seminales de Schmalensee (1985) y Rumelt (1991) sobre evaluación del desempeño empresarial, recogen en un estudio empírico realizado en cooperativas de trabajo asociado y sociedades laborales en la Comunidad Valenciana 
el resultado de los efectos que estos autores denominan "empresa de economía social general" y "empresa de economía social sectorializado".

En los resultados de dicho estudio no se distinguen de una forma significativa ambos efectos, por lo que se pueden aplicar criterios comunes de valoración del desempeño a diferentes tipos de empresas, sin ser significativas las diferencias sectoriales. Estos autores proponen un conjunto de variables proxy ${ }^{10}$ para medir la "performance" o desempeño empresarial.

Dada la reducida dimensión de este tipo de empresas, y la falta de medios para registrar medidas de desempeño de cierta calidad informativa, se ha optado por establecer unos indicadores en la línea del planteamiento anterior, algo más subjetivos, aunque no por ello menos fiables.

Apoyando esto, la literatura empírica reporta una gran diversidad de indicadores de desempeño, realizando una distinción común entre medidas financieras y no financieras. Las no financieras incluyen objetivos como la satisfacción y calificaciones de éxito global hechas por los propietarios y gerentes de negocios (Rauch et al., 2009).

En el presente trabajo se han considerado los siguientes indicadores para medir este constructo, valorados mediante una escala de Likert de 1-5, siendo 1 el valor mínimo en cuanto evolución o valoración, y 5 el valor máximo:

- Y11: Evolución de la cifra de negocio.

- Y12: Evolución o crecimiento de la plantilla (socios y no socios).

- Y13: Evolución o crecimiento de las inversiones.

- Y14: Valoración del desempeño global del negocio.

- Y15: Valoración del desempeño en el último año.

- Y16: Valoración del desempeño con relación a competidores directos.

Los tres primeros indicadores establecen respectivamente la evolución comparativa entre el inicio de la actividad y el momento actual de tres aspectos clave de la empresa: la cifra de negocio, el nivel de empleo y el esfuerzo inversor. Dichos indicadores son objetivos, al responder a valores cuantitativos comprobables, mientras que los tres últimos indicadores, relativos a la valoración del desempeño, corresponden más a las calificaciones o percepciones subjetivas de los directivos, aunque en buena medida son verificables.

10.- Un conjunto formado por 13 variables, integradas en dos ejes. El primero de ellos evalúa el sistema productivo con información cuantitativa sobre el consumo de inputs y la productividad obtenida. El segundo eje agrupa variables relativas al tamaño, trayectoria, inversiones y rentabilidad empresarial. 


\subsubsection{Resultados personales (RESPER)}

Los distintos elementos que afectan a las expectativas de los emprendedores pueden agruparse en dos tipos de mecanismos básicos que actúan sobre la motivación humana: mecanismos de motivación extrínseca y de motivación intrínseca (Orellana y Monreal, 2007), que están en estrecha relación con el diseño de incentivos.

La motivación será extrínseca cuando el comportamiento de una persona al realizar una determinada tarea está determinado en gran parte por el beneficio que se espera obtener, o por pérdidas que se desea evitar, siendo esas ganancias o pérdidas fundamentalmente de naturaleza material, y en estrecha relación con los incentivos monetarios, o de naturaleza material.

En cambio, la motivación será intrínseca cuando la realización de la tarea es motivadora en sí misma, es decir, cuando la persona no necesita de estímulos externos para realizar la tarea de acuerdo a lo pactado. Son múltiples las formas y situaciones en las que las personas en la empresa pueden sentirse motivadas intrínsecamente, por ejemplo, cuando se les asigna tareas que resultan muy gratificantes de realizar, tienen la posibilidad de participar en las decisiones, o comparten la propiedad de la empresa, como es el caso de los socios-trabajadores en la CTA. En este sentido y relacionado con el emprendimiento, cabe destacar las investigaciones recientes de Chen,Chang, y Lin (2018); Shepherd y Patzelt (2018); y O'Shea, Buckley, y Halbesleben (2017) que revelan que las personas pueden sentirse motivadas a emprender por factores intrínsecos como la felicidad o el bienestar emocional.

En base a las características propias de la CTA, y sin ser exhaustivos en la selección de criterios ${ }^{11}$, se ha establecido un conjunto de indicadores, escogidos con un criterio simplificador, para medir el constructo reflectivo resultados personales, considerando el nivel medio de logro o grado de satisfacción alcanzado en las expectativas de todos los emprendedores, con una escala de Likert 1-5, en relación con la motivación intrínseca y la motivación extrínseca (siendo 1: un nivel mínimo de logro, y 5: un nivel máximo):

- Y21: Relaciones satisfactorias con socios/compañeros.

- Y22: Satisfacción con el trabajo realizado.

- Y23: Aceptación de sus ideas por los demás.

- Y24: Cumplimiento de expectativas laborales

- Y25: Complimiento de expectativas profesionales y empresariales.

11.- Ya que, por ejemplo, la satisfacción por la participación en la toma de decisiones, o la satisfacción por compartir la propiedad de la empresa, se dan por hecho en la cooperativa. Tan sólo han de considerarse aquellos factores de motivación intrínseca y extrínseca que, sin ser propios de las cooperativas y no de carácter más bien invariante, se pueda apreciar una variabilidad de ellos en las diferentes cooperativas, debido a la influencia de diferentes factores. 
Los tres primeros indicadores miden aspectos de la motivación intrínseca, en estrecha relación con aquellos dirigidos hacia el clima laboral, las condiciones de trabajo y las expectativas personales. Los dos restantes miden aspectos de motivación extrínseca, y se refieren al nivel de cumplimento de sus expectativas desde una perspectiva material, 0 al menos con una mayor aportación o participación de estímulos externos, como son las expectativas laborales, profesionales y empresariales.

\subsection{Modelo estructural. Relaciones e hipótesis}

Los resultados del emprendimiento en las CTA, como se ha establecido anteriormente, se representan en dos constructos, medidos cada uno por sus propios indicadores, correspondiendo el primero a los resultados o la performance de la empresa (DESEMP), obtenidos por el desempeño de los socios y trabajadores en la misma, siendo estos resultados de carácter material; y el segundo a los resultados de carácter más cualitativo, derivados del nivel de cumplimiento de sus expectativas personales, profesionales y empresariales (RESPER).

Para las variables del resultado, y desde una perspectiva multidimensional, se trata de determinar cómo el grado de experiencia de los promotores, la capacidad de establecer relaciones provechosas con los agentes del entorno, el grado de atractivo del sector elegido para emprender, la efectividad de los mecanismos y órganos de control que la cooperativa ha establecido, el origen y las causas o razones de los emprendedores para tomar la decisión de emprender, e incluso el efecto de ciertas variables demográficas como son la edad, el género o el nivel educativo de los emprendedores, todo ello puede influir en los niveles de rendimiento o performance de la empresa, por un lado, y por otro actuar sobre el nivel de cumplimento de los resultados personales, afectando al comportamiento de los miembros de la cooperativa de trabajo, a sus expectativas y su sistema de incentivos, en los aspectos más cualitativos.

Por otra parte, se establece la hipótesis de que los niveles de desempeño DESEMP) obtenidos median en la relación o influencia que los factores independientes tienen sobre las expectativas 0 resultados personales (RESPER). Es decir, se trata de medir la influencia indirecta de una variable de resultado sobre la otra.

Todo esto da lugar a las hipótesis teóricas que con carácter general han sido planteadas en el apartado 2 , y que a continuación van a ser contrastadas en el modelo estructural propuesto. Las relaciones establecidas han sido modelizadas con la metodología de ecuaciones estructurales PLS-SEM, obteniendo los correspondientes coeficientes de regresión ß-estandarizados y su significatividad estadística, que evalúan la importancia de la relación entre dichos factores.

El grado de significatividad de las relaciones se obtiene mediante los valores t-Student, a través de bootstrapping, y también por la técnica de intervalos de confianza (Preacher y Hayes, 2008; Hayes (2009). Se obtienen los índices $R^{2}, Q^{2}$ y AVE (varianza extraída). Se determinan las propiedades psi- 
cométricas de los modelos de medida y estructural, en cuanto a su fiabilidad compuesta y su validez, tanto convergente como discriminante, realizándose el oportuno contraste de hipótesis.

La figura 3 representa el modelo estructural, que recoge las relaciones directas entre factores independientes y los factores del resultado emprendedor, así como las relaciones de mediación descritas.

\section{Figura 3. Modelo estructural del proceso de emprendimiento en la CTA}

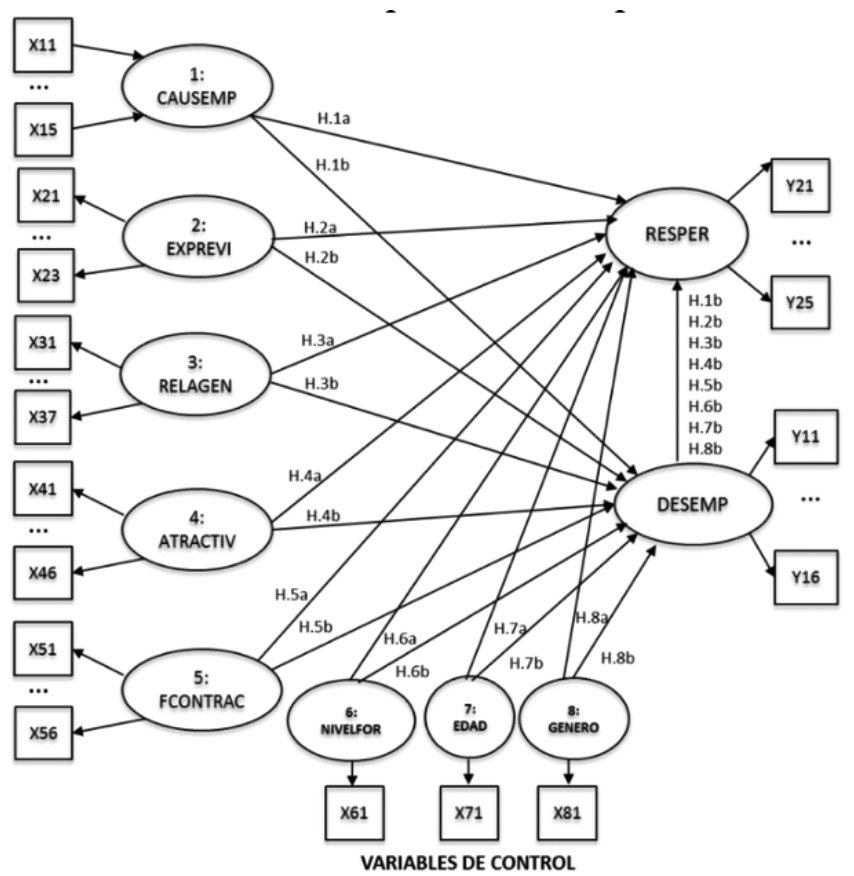

FUENTE: Elaboración propia.

La hipótesis que se establecen en el modelo estructural, son las siguientes:

Hipótesis H. 1 a: El factor causas para el emprendimiento (CAUSAEMP) tiene una influencia directa sobre el factor resultados personales (RESPER).

Hipótesis $H_{1}$ b: El factor causas para el emprendimiento (CAUSAEMP) tiene una influencia directa sobre el factor desempeño o performance de la empresa (DESEMP), e indirecta sobre los resultados personales (RESPER), a través de la mediación del desempeño (DESEMP). 
Hipótesis $H_{.2}$ a: El factor experiencia previa (EXPREVI) tiene una influencia directa sobre el factor resultados personales (RESPER).

Hipótesis $H_{2} 2$ b: El factor experiencia previa (EXPREVI) tiene una influencia directa sobre el factor desempeño o performance de la empresa (DESEMP), e indirecta sobre los resultados personales (RESPER), a través de la mediación del desempeño (DESEMP).

Hipótesis H. 3 a: El factor relaciones con los agentes del entorno (RELAGEN) tiene una influencia directa sobre el factor resultados personales (RESPER).

Hipótesis $\mathrm{H}_{3}$ b: El factor relaciones con los agentes del entorno (RELAGEN) tiene una influencia directa sobre el factor desempeño o performance de la empresa (DESEMP), e indirecta sobre los resultados personales (RESPER), a través de la mediación del desempeño (DESEMP).

Hipótesis $\mathrm{H}_{4}$ a: El factor atractivo del sector (ATRACTIV) tiene una influencia directa sobre el factor resultados personales (RESPER).

Hipótesis $\mathrm{H}_{4}$ b: El factor atractivo del sector (ATRACTIV) tiene una influencia directa sobre el factor desempeño o performance de la empresa (DESEMP), e indirecta sobre los resultados personales (RESPER), a través de la mediación del desempeño (DESEMP).

Hipótesis $\mathrm{H}_{.}$a: El factor forma contractual (FCONTRAC) tiene una influencia directa sobre el factor resultados personales (RESPER).

Hipótesis $\mathrm{H}_{5} \mathrm{~b}_{\mathrm{b}}$ : El factor forma contractual (FCONTRAC) tiene una influencia directa sobre el factor desempeño o performance de la empresa (DESEMP), e indirecta sobre los resultados personales (RESPER), a través de la mediación del desempeño (DESEMP).

Hipótesis $\mathrm{H}_{6} 6$ a: El factor nivel formativo (NIVELFOR) tiene una influencia directa sobre el factor resultados personales (RESPER).

Hipótesis $\mathrm{H}_{.} 6$ b: El factor formativo (NIVELFOR) tiene una influencia directa sobre el factor desempeño o performance de la empresa (DESEMP), e indirecta sobre los resultados personales (RESPER), a través de la mediación del desempeño (DESEMP).

Hipótesis $H$. 7 a: El factor edad (EDAD) tiene una influencia directa sobre el factor resultados personales (RESPER).

Hipótesis $H .7$ b: El factor edad (EDAD) tiene una influencia directa sobre el factor desempeño o performance de la empresa (DESEMP), e indirecta sobre los resultados personales (RESPER), a través de la mediación del desempeño (DESEMP).

Hipótesis $H_{.8}$ a: El factor género (GENERO) tiene una influencia directa sobre el factor resultados personales (RESPER).

Hipótesis $H_{.8} b$ : El factor género (GENERO) tiene una influencia directa sobre el factor desempeño o performance de la empresa (DESEMP), e indirecta sobre los resultados personales (RESPER), a través de la mediación del desempeño (DESEMP). 


\section{Análisis y discusión de resultados}

\subsection{Evaluación del modelo de medida}

Los cuadros 4 y 5 reportan los resultados que evalúan la fiabilidad y la validez del modelo estructural. El cuadro 4 muestra que todos los factores del modelo tienen un alto grado de fiabilidad compuesta, con valores requeridos superiores a 0'7, y los ítems que han permanecido tras la depuración están muy correlacionados y representan fielmente a cada factor; al igual que su validez convergente, con valores de varianza extraída (AVE) por encima de 0'5.

El cuadro 5 muestra la validez discriminante de los diferentes constructos, al ser la raíz cuadrada de su AVE superior a las correlaciones con el resto de factores, lo que indica que las escalas miden adecuadamente cada una a su constructo, y no a otra cosa.

\section{Cuadro 4. Fiabilidad y validez convergente del instrumento de medida}

\begin{tabular}{|lcccc|}
\hline Variable latente & $\begin{array}{c}\text { Fiabilidad } \\
\text { compuesta }\end{array}$ & $\begin{array}{c}\text { AVE } \\
\text { (varianza extraída promedio) }\end{array}$ & $\mathbf{R}^{\mathbf{2}}$ & $\mathbf{Q}^{\mathbf{2}}$ \\
\hline CAUSEMP & - & - & & \\
EXPREVI & 0,7234 & 0,5689 & & \\
RELAGEN & 0,8719 & 0,6302 & & \\
ATRACTIV & 0,8185 & 0,6932 & & \\
FCONTRAC & 0,8987 & 0,7505 & & \\
NIVELFOR & 1,0000 & 1,0000 & & \\
EDAD & 1,0000 & 1,0000 & & \\
GENERO & 1,0000 & 1,0000 & & \\
DESEMP & 0,9510 & 0,8297 & 0,5935 & 0,4928 \\
RESPER & 0,9627 & 0,8378 & 0,8979 & 0,7466 \\
\hline
\end{tabular}




\section{Cuadro 5. Validez discriminante del instrumento de medida}

\begin{tabular}{|lcccccccccc|}
\hline Variable latente & 1 & 2 & 3 & 4 & 5 & 6 & 7 & 8 & 9 & 10 \\
\hline 1: CAUSEMP & - & & & & & & & & & \\
2: EXPREVI & - & 0,7543 & & & & & & & & \\
3: RELAGEN & - & 0,5368 & $\mathbf{0 , 7 9 3 9}$ & & & & & & & \\
4: ATRACTIV & - & 0,4214 & 0,5152 & 0,8326 & & & & & & \\
5: FCONTRAC & - & 0,4956 & 0,8422 & 0,5927 & 0,8663 & & & & & \\
6: NIVELFOR & - & 0,1103 & 0,4526 & 0,4155 & 0,4419 & 1,0000 & & & & \\
7: EDAD & - & 0,2243 & 0,2419 & 0,2704 & 0,2314 & 0,4649 & 1,0000 & & & \\
8: GENERO & - & 0,3932 & 0,4147 & 0,3696 & 0,4322 & 0,1468 & 0,1873 & 1,0000 & & \\
9: DESEMP & - & 0,5223 & 0,5052 & 0,5003 & 0,4218 & 0,2835 & 0,1096 & 0,4314 & 0,9109 & \\
10: RESPER & - & 0,5839 & 0,6559 & 0,5268 & 0,5242 & 0,4410 & 0,0516 & 0,5525 & 0,8762 & 0,9153 \\
\hline
\end{tabular}

Diagonal: Raíz cuadrada de la Varianza Extraída Promedio (AVE)

Triángulo inferior: correlaciones entre las variables latentes

El factor causas para el emprendimiento (CAUSEMP), por tratarse de un constructo formativo, no tiene sentido en él el uso los anteriores indicadores, requiriendo otro tipo de análisis. En efecto, no tiene sentido en él los conceptos de fiabilidad y validez.

Por el contrario, es importante que no existan problemas de multicolinealidad entre sus ítems, porque de ser así sería difícil discernir el efecto individual de cada uno sobre el factor. Para averiguar la existencia de multicolinealidad se ha utilizado el indicador VIF (factor de inflación de la varianza), calculado con SPSS, habiéndose obtenido que todos los ítems del constructo la VIF son inferiores al valor de 5, lo que indica ausencia de multicolinealidad (Mason y Perreault, 1991). 
4.2. Evaluación del modelo estructural y contraste de hipótesis

Cuadro 6. Modelo estructural - contraste de hipótesis

\begin{tabular}{|c|c|c|c|c|c|}
\hline \multirow[t]{2}{*}{ Hipótesis } & \multirow[t]{2}{*}{ Relación } & \multirow[t]{2}{*}{$\beta$ estandarizado } & \multirow[t]{2}{*}{ Valor $\mathrm{t}$} & \multicolumn{2}{|c|}{ Intervalo de confianza } \\
\hline & & & & Percentil 0,025 & Percentil 0,975 \\
\hline \multirow[t]{2}{*}{$\mathrm{H} .1 \mathrm{~b}$} & CAUSEMP $\rightarrow$ DESEMP & $-0,0302$ & 0,2517 & $-0,2544$ & 0,2098 \\
\hline & & & no significativo & & \\
\hline H.1a & CAUSEMP $\rightarrow$ RESPER & 0,1516 & $2,2836^{*}$ & 0,0004 & 0,2641 \\
\hline H.2b & EXPREVI $\rightarrow$ DESEMP & 0,3832 & $2,8555^{* *}$ & 0,0333 & 0,5680 \\
\hline \multirow[t]{2}{*}{ H.2a } & EXPREVI $\rightarrow$ RESPER & 0,0975 & 1,5035 & $-0,0623$ & 0,1932 \\
\hline & & & no significativo & & \\
\hline H.3b & RELAGEN $\rightarrow$ DESEMP & 0,3024 & $2,2669^{*}$ & 0,0921 & 0,6288 \\
\hline H.3a & RELAGEN $\rightarrow$ RESPER & 0.2563 & $2,8876^{* *}$ & 0,1159 & 0,4585 \\
\hline H. $4 \mathrm{~b}$ & ATRACTIV $\rightarrow$ DESEMP & 0,2879 & $4,0621^{\star \star \star}$ & 0,1498 & 0,4235 \\
\hline \multirow[t]{2}{*}{ H.4a } & ATRACTIV $\rightarrow$ RESPER & $-0,0117$ & 0,2578 & $-0,1000$ & 0,0798 \\
\hline & & & no significativo & & \\
\hline H. $5 \mathrm{~b}$ & FCONTRAC $\rightarrow$ DESEMP & 0,3208 & $2,4230^{*}$ & 0,0631 & 0,2701 \\
\hline H.5a & FCONTRAC $\rightarrow$ RESPER & 0,1556 & $2,2233^{*}$ & 0,0274 & 0,3151 \\
\hline H.6b & NIVELFOR $\rightarrow$ DESEMP & 0,3073 & $2,6567^{\star *}$ & 0,0536 & 0,4168 \\
\hline H.6a & NIVELFOR $\rightarrow$ RESPER & 0,2139 & $3,1305^{\star *}$ & 0,0334 & 0,3058 \\
\hline $\mathrm{H} .7 \mathrm{~b}$ & EDAD $\rightarrow$ DESEMP & 0,4510 & $4,5897^{\star \star \star}$ & 0,2365 & 0,6214 \\
\hline \multirow[t]{2}{*}{ H.7a } & EDAD $\rightarrow$ RESPER & 0.1011 & 1,8773 & $-0,0331$ & 0,1780 \\
\hline & & & no significativo & & \\
\hline H. $8 \mathrm{~b}$ & GENERO $\rightarrow$ DESEMP & 0,2396 & $1,9870^{*}$ & 0,0132 & 0,4816 \\
\hline H.8a & GENERO $\rightarrow$ RESPER & 0,1487 & $2,7736^{* *}$ & 0,0357 & 0,2443 \\
\hline H.1a .. H.8a & DESEMP $\rightarrow$ RESPER & 0,5910 & $9,0465^{\star \star *}$ & 0,4172 & 0,6891 \\
\hline
\end{tabular}

El cuadro 4 reporta también los resultados para poder evaluar la calidad del modelo en cuanto a su poder y su capacidad predictivos mediante los indicadores $R^{2}$ y $Q^{2}$. El primero de ellos, según Ios niveles sugeridos por Chin (1998) indica que el modelo estructural tiene un poder predictivo moderado para el factor DESEMP $\left(R^{2}=0,5935\right)$, y fuerte para el factor $R E S P E R\left(R^{2}=0,8979\right)$.

Por su parte, el valor positivo de $Q^{2}$ indica que el modelo tiene capacidad predictiva para ambos factores de resultado $\left(Q^{2}=0,4928\right.$ y $Q^{2}=07466$, respectivamente).

El contraste de hipótesis se realiza mediante dos métodos: a) proceso bootstrapping de PLS, obteniendo el valor de t-Student que determina el grado de significatividad estadística; y b) intervalos de confianza para percentiles 0'025 y 0'975, una técnica no paramétrica que no depende de ninguna dis- 
tribución (Preacher y Hayes, 2008; Hayes (2009), según la cual, cuando la relación que se mide es significativa, el intervalo de confianza no debe incluir el valor 0 . Ambos resultados se reportan en el cuadro 6 , siendo coincidentes en todos los casos.

En una primera aproximación a la interpretación de los resultados obtenidos y de cuáles son las hipótesis que se cumplen, y cuáles no (cuadro 6), se puede establecer que:

- Causas para el emprendimiento (CAUSEMP) es el único factor que influye de forma directa y significativa sobre los resultados personales $\left(\beta=0^{\prime} 1516\right)$, sin ningún tipo de impacto 0 influencia sobre el desempeño ( $\beta=-0^{\prime} 0302$ ). Esto se interpreta como que la diferente causa u origen de los emprendedores no supone una mayor incidencia en los niveles de rendimiento o desempeño en la empresa, y sin embargo es un factor que afecta a los aspectos más cualitativos de los emprendedores.

Por el contrario, experiencia previa (EXPREVI) y atractivo del sector (ATRACTIV) afectan únicamente al desempeño y a los niveles de performance en la empresa $(\beta=0$ '3832 y $\beta=0$ '2879 respectivamente), sin ninguna influencia significativa en las expectativas o resultados personales. Al parecer, la experiencia laboral o profesional anterior se plasma más en los resultados de la empresa, sin que tenga una influencia significativa en los aspectos personales de los emprendedores. Aún con mayor razón, cabe pensar que el atractivo del sector, un factor considerado pull o de oportunidad, afecta tan sólo, o fundamentalmente, a los niveles de performance.

- Relaciones con agentes del entorno (RELAGEN) influye directa y significativamente sobre ambos factores del resultado ( $\beta=0$ '3024 y $\beta=0$ '2563), que representan relaciones de índole técnica, asesoramiento, colaboración e intercambio y relaciones de carácter institucional. Son relaciones de carácter estable establecidas con dichos agentes, con el objetivo de la mejora del desempeño y la performance de la empresa, lo que explica el importante impacto sobre esa variable de resultado.

Además, el establecimiento de relaciones institucionales mejora sin duda el grado de satisfacción personal y las expectativas de los socios-trabajadores en diferentes aspectos, como sentirse representados, asesorados, apoyados e incluso escuchados por parte de dichos agentes, públicos y privados.

- El nivel formativo (NIVELFOR) tiene una influencia o impacto sobre ambas variables del resultado, sobre todo en los niveles de desempeño o performance de la empresa ( $\left.\beta=0^{\prime} 3073\right)$, y en menor medida en los resultados personales $\left(\beta=0^{\prime} 2139\right)$. En el primer caso, la relación es obvia al entender que la mayor cualificación técnica y profesional de los emprendedores conduce a mayores niveles de desempeño en su empresa, y en cuanto al segundo caso, la cooperativa supone un estímulo y una recompensa personal a sus esfuerzos de formación, que colma sus expectativas personales.

Por su parte, el factor Forma contractual (FCONTRAC) tiene también una influencia directa sobre los dos tipos del resultado emprendedor, con una mayor incidencia en el desempeño o la performance 
la empresa $(\beta=0$ '3208), y algo más atenuada sobre los resultados personales de los emprendedores cooperativos $\left(\beta=0^{\prime} 1556\right)$.

Esto puede interpretarse para DESEMP como el impacto o efecto en el desempeño de los mecanismos de control y de otros aspectos organizativos implantados en la empresa, relacionados con la peculiar relación circular de agencia en este tipo de organizaciones (Orellana y Monreal, 2007).

En cuanto a RESPER, destacan aquí los aspectos cualitativos y la influencia que ejerce este factor sobre el comportamiento y el nivel de expectativas personales de los miembros que participan en el proyecto emprendedor, dadas las características de sus relaciones laborales y la participación en las decisiones y en la propiedad en este tipo de organización.

Por último, en cuanto a la relación establecida entre las dos variables de resultado, en la que DESEMP actúa como variable independiente, e influye sobre RESPER, se observa un importante coeficiente de regresión ( $\beta=0^{\prime} 5910$ ), afectando dicha relación en los efectos de mediación de DESEMP que a continuación se van a exponer.

\subsection{Análisis de la mediación de la variable desempeño sobre la variable resulta- dos personales}

Los resultados se reportan de acuerdo con el enfoque más respaldado actualmente (Preacher y Hayes, 2008; Hayes (2009), que recomienda un test de mediación no enfocado en los caminos individuales sino en el término del producto $a^{*} b$, a partir de técnicas no paramétricas, basadas en la utilización de intervalos de confianza para percentiles 0'025 y 0'975, según el cual, si existe mediación, el intervalo del efecto $a^{*} b$ no debe incluir el valor 0 .

El cuadro 7 recoge los resultados del análisis de la mediación, que confirman que existe un efecto de mediación del factor DESEMP en la relación indirecta entre los factores independientes, incluidas las variables de control, y RESPER, siendo significativa dicha relación de mediación, con excepción del factor causas para el emprendimiento (CAUSEMP). Eso es así porque al no ser ya significativa la relación CAUSEMP $\rightarrow$ DESEMP, tampoco puede serlo la relación CAUSEMP $\rightarrow$ DESEMP $\rightarrow$ RESPER. Para el resto de factores, al ser significativa su relación directa con DESEMP, sí se verificará dicha mediación. 
Cuadro 7. Intervalo de confianza de los efectos mediadores

\begin{tabular}{|lccc|}
\hline Relación & Percentil 0,025 & Percentil 0,975 & observaciones \\
\hline CAUSEMP $\rightarrow$ DESEMP $\rightarrow$ RESPER & $-0,1530$ & 0,1174 & No significativo \\
EXPREVI $\rightarrow$ DESEMP $\rightarrow$ RESPER & 0,0174 & 0,3443 & \\
RELAGEN $\rightarrow$ DESEMP $\rightarrow$ RESPER & 0,0544 & 0,3601 & \\
ATRACTIV $\rightarrow$ DESEMP $\rightarrow$ RESPER & 0,0772 & 0,2629 & \\
FCONTRAC $\rightarrow$ DESEMP $\rightarrow$ RESPER & 0,0727 & 0,3262 & \\
NIVELFOR $\rightarrow$ DESEMP $\rightarrow$ RESPER & 0,0238 & 0,2563 & \\
EDAD $\rightarrow$ DESEMP $\rightarrow$ RESPER & 0,1126 & 0,3777 & \\
GENERO $\rightarrow$ DESEMP $\rightarrow$ RESPER & 0,0072 & 0,2879 & \\
\hline
\end{tabular}

El análisis de la mediación, se completa con el cálculo de indicador VAF, o Variance Accounted For (Hair et al., 2014), como muestra el cuadro 8, para cuantificar y tipificar dicha mediación, siendo los valores de referencia: $\mathrm{VAF}<0$ '20: no existe mediación; 0'20<VAF<0'80: la mediación es parcial, teniendo la variable independiente todavía un efecto directo significativo sobre la dependiente; y VAF>0'80: mediación total, cuando la variable independiente no tenía ningún efecto directo sobre la variable dependiente, y al incluir en el modelo a la variable mediadora se restablece la relación.

\section{Cuadro 8. Tipos de mediación - cálculo de la VAF}

\begin{tabular}{|l|cccccc|}
\hline Relación & $\begin{array}{c}\beta \text { estandari- } \\
\text { zado }\end{array}$ & $\begin{array}{c}\text { Efecto } \\
\text { directo } \\
\text { factor } \rightarrow \\
\text { RESPER }\end{array}$ & $\begin{array}{c}\text { Efecto } \\
\text { indirecto } \\
\text { factor } \rightarrow \text { DESEMP } \\
\rightarrow \text { RESPER }\end{array}$ & $\begin{array}{c}\text { Efecto } \\
\text { total }\end{array}$ & $\begin{array}{c}\text { VAF } \\
\text { Variance } \\
\text { Accounted } \\
\text { For }\end{array}$ & $\begin{array}{c}\text { Tipo de } \\
\text { mediación }\end{array}$ \\
\hline $\begin{array}{l}\text { EXPREVI } \rightarrow \text { DESEMP } \\
\text { EXPREVI } \rightarrow \text { RESPER }\end{array}$ & $\begin{array}{c}0,3832^{* *} \\
0,0975 \mathrm{~ns}\end{array}$ & 0,0975 & 0,2265 & 0,3240 & 0,6990 & Parcial/total \\
RELAGEN $\rightarrow$ DESEMP & $0,3024^{*}$ & & & & & \\
RELAGEN $\rightarrow$ RESPER & $0,2563^{* *}$ & 0,2563 & 0,1787 & 0,4350 & 0,4108 & Parcial \\
ATRACTIV $\rightarrow$ DESEMP & $0,2879^{* *}$ & & & & & \\
ATRACTIV $\rightarrow$ RESPER & $0,0117 \mathrm{~ns}$ & 0,0117 & 0,1701 & 0,1818 & 0,9357 & Total \\
FCONTRAC $\rightarrow$ DESEMP & $0,3208^{*}$ & & & & & \\
FCONTRAC $\rightarrow$ RESPER & $0,1556^{*}$ & 0,1556 & 0,1896 & 0,3452 & 0,5492 & Parcial \\
NIVELFOR $\rightarrow$ DESEMP & $0,3073^{* *}$ & & & & & \\
NIVELFOR $\rightarrow$ RESPER & $0,2139^{* *}$ & 0,2139 & 0,1264 & 0,3403 & 0,3714 & Parcial \\
EDAD $\rightarrow$ DESEMP & $0,4510^{* *}$ & & & & & \\
EDAD $\rightarrow$ RESPER & $0,1011 \mathrm{~ns}$ & 0,1011 & 0,2665 & 0,3676 & 0,7250 & Parcial/total \\
GENERO $\rightarrow$ DESEMP & $0,2396^{*}$ & & & & & \\
GENERO $\rightarrow$ RESPER & $0,1487^{* *}$ & 0,1487 & 0,1416 & 0,2903 & 0,4878 & Parcial \\
\hline
\end{tabular}

ns: no significativo ${ }^{* * *} \mathrm{p}<0,001^{* *} \mathrm{p}<0,01{ }^{*} \mathrm{p}<0,05$

CIRIEC-España, Revista de Economía Pública, Social y Cooperativa 
A la vista de los resultados obtenidos en los factores que no tienen una influencia directa y significativa en los resultados personales, como ocurre con la experiencia previa (EXPREVI), el atractivo del sector (ATRACTIV) y la edad (EDAD), con unos valores muy altos de VAF, esto confirma el importante efecto de mediación de la variable desempeño o performance (DESEMP) sobre los resultados personales (RESPER), para que finalmente dichos factores independientes afecten a este último factor. Para el resto de factores, con efectos directos y significativos sobre ambos factores de resultado, la mediación es de tipo parcial, y menor es el valor de la VAF resultante.

\subsection{Papel de las variables demográficas de control}

Al incorporar en el modelo estructural las variables demográficas, éstas contribuyen conjuntamente a la $\mathrm{R}^{2}$ de las variables dependientes, y conviene realizar la prueba del efecto tamaño, effect size 0 prueba $f^{2}$ (Cohen 1998), para explorar aquellos cambios producidos en dicho indicador cuando se incluye o elimina una determinada variable de control del modelo, determinando así la relevancia predictiva de esa variable (Chin, 1998). Los niveles de referencia de $f^{2}$ para establecer la relevancia predictiva del modelo son los siguientes: 0 '02 $<f^{2}<0$ '15: relevancia predictiva pequeña o débil; 0.15 $<f^{2}<0.35$ : relevancia predictiva mediana o moderada; y $f^{2}>0$ '35: relevancia predictiva amplia 0 fuerte.

El cuadro 9 recoge los resultados de $f^{2}$, excluyendo sucesivamente del modelo a cada una de las variables de control, o bien a todas ellas conjuntamente, para obtener los valores de $\mathrm{R}^{2}$ excluida, y hacer los cálculos de $f^{2}$ con respecto a la $\mathrm{R}^{2}$ del modelo original $\left(\mathrm{R}^{2}\right.$ incluida), según la siguiente expresión: $f^{2}=\left(R^{2}\right.$ incluida $-R^{2}$ excluida $) / 1-R^{2}$ incluida.

\section{Cuadro 9. Relevancia predictiva de las variables de control (prueba f2)}

\begin{tabular}{|c|c|c|c|c|c|c|c|c|c|}
\hline & \multicolumn{5}{|c|}{$R^{2}$} & \multicolumn{4}{|c|}{$t^{2}$} \\
\hline VARIABLE & & NIVEL & & & TODAS & NIVEL & & & TODAS \\
\hline DEPENDIENTE & ORIGINAL & FORMAT & EDAD & GENERO & CONJUNT. & FORMAT & EDAD & GENERO & CONJUNT. \\
\hline RESPER & 0,8979 & 0,875 & 0,892 & 0,886 & 0,870 & 0,22 & 0,06 & 0,12 & 0,27 \\
\hline DESEMP & 0,5935 & 0,519 & 0,447 & 0,555 & 0,409 & 0,18 & 0,36 & 0,09 & 0,45 \\
\hline
\end{tabular}

Los resultados muestran una relevancia predictiva intermedia o moderada para todas variables de control consideradas en su conjunto, con respecto a la variable RESPER $\left(f^{2}=0,27\right)$, y una relevancia amplia o fuerte con respecto a DESEMP $\left(f^{2}=0,45\right)$. 
En cuanto al papel individual de cada variable de control, destaca la influencia del nivel formativo sobre DESEMP $\left(f^{2}=0,18\right)$ y RESPER $\left(f^{2}=0,22\right)$, ambos con una relevancia predictiva moderada, y la edad sobre DESEMP, con un valor de $f^{2}=0,36$ (relevancia predictiva fuerte). Para el resto de casos, el impacto de las variables de control sobre las variables dependientes es menor, con una relevancia predictiva débil.

Estos resultados confirman en gran medida los obtenidos en el contraste de hipótesis. En ese sentido, el nivel formativo está presente tanto en los niveles de desempeño de la empresa como en los resultados personales. Por su lado, la edad es claramente un factor de empuje que incide más en la performance de la empresa y apenas lo hace en los resultados personales. El género, sin embargo, y a pesar de ser significativas sus relaciones con ambas variables del resultado emprendedor, en realidad se comprueba su débil relevancia predictiva para dichas variables de resultado $\left(f^{2}=0,12\right.$ y $\left.f^{2}=0,09\right)$.

\subsection{Análisis de la moderación e interacción entre factores}

La moderación de una variable o factor sobre la relación existente entre otras variables es un fenómeno estudiado para comprobar y analizar los efectos que produce dicha moderación o interacción en una relación causal existente. Por definición, un efecto moderador se produce cuando una variable (moderadora) modifica la fuerza (valor de b estandarizado) o la dirección (signo) entre una variable predictora y una variable dependiente.

Entre los enfoques para estimar el efecto moderador, el más reconocido es aquel que testea el producto de las variables, estandarizando éstas y creando una nueva que resulta del producto de las variables predictora $(\mathrm{X})$ y moderadora $(\mathrm{M})$. La hipótesis de moderación plantea que el path de la interacción $(X, M)$ sobre $Y$ ha de ser significativo, para lo cual se analizan posibles situaciones en las que una variable exógena puede interaccionar con otra variable exógena, en su relación con una variable dependiente.

La forma de establecer dicha interacción la proporciona PLS-SEM, creando una nueva variable estandarizada, tipo producto ( $\mathrm{X}, \mathrm{M})$, que calcula el coeficiente de regresión (b estandarizado) y la dirección (signo) de la interacción, así como la significatividad de dicha relación. La evaluación del efecto moderador se realiza explorando los valores de $\mathrm{R}^{2}$ en el modelo original y en el modelo con interacción, con la prueba $f^{2}$ mediante la expresión: $f^{2}=\left(R^{2}\right.$ con interacción $-R^{2}$ sin interacción $) / 1-R^{2}$ con interacción, con los valores de referencia (Cohen, 1998; Chin 1998): por debajo de 0'02 no existe moderación; hasta 0,15 el efecto moderador es débil; hasta 0'35 el efecto es moderado, y en adelante la moderación es fuerte o importante.

En el conjunto de variables analizadas, se comprueba la existencia de una interacción o efecto de moderación que produce la variable EXPREVI en la relación entre la variable independiente RELA- 
GEN y la variable dependiente DESEMP. Es decir, la experiencia previa de tipo laboral, profesional o empresarial de los emprendedores modera en la relación o influencia que ejerce el establecimiento de relaciones con agentes del entorno sobre los niveles de resultados o performance de la empresa. El cuadro 10 reporta los resultados de dicha moderación.

El valor de $f^{2}=0$ ' 18 indica la existencia de un efecto moderador de tipo intermedio o moderado. Por otra parte, el signo negativo del path de la interacción $(-0,464)$, y el menor valor del coeficiente de regresión de la predictora RELAGEN en el modelo con moderación ( $\beta=0$ '1591) con respecto al modelo original $(\beta=0$ '3024), indica un efecto de atenuación producido por la variable moderadora EXPREVI en la influencia que la variable predictora RELAGEN ejerce sobre la variable de resultado DESEMP.

\section{Cuadro 10. Efecto moderador de la experiencia previa sobre la influencia de las relaciones con los agentes del entorno en el desempeño o performance de la empresa}

\begin{tabular}{|lc|}
\hline Variable predictora: RELAGEN & Variable dependiente: \\
Variable moderadora: EXPREVI & DESEMP \\
\hline B variable predictora original & 0,3024 \\
B variable moderadora original & 0,3832 \\
B variable predictora con interacción & 0,1591 \\
B variable moderadora con interacción & 0,3002 \\
B interacción & $-0,464$ \\
20t-Student interacción & $2,317^{\star}$ \\
Valor de $f^{2}$ & 0,18 \\
\hline
\end{tabular}

${ }^{* \star *} p<0,001{ }^{* *} p<0,01{ }^{*} p<0,05$

Aunque sin duda, para una adecuada interpretación de los efectos de moderación se recomienda utilizar la técnica Johnson-Neyman (Johnson y Neyman, 1936; Pothoff, 1964; Bauer y Curran, 2005), basada en pruebas de pendiente simples (Cohen et al., 2003), para evaluar si la relación (pendiente) entre $\mathrm{X}$ e $\mathrm{Y}$ es significativa para un valor particular de $\mathrm{M}$, identificando regiones en el rango de la variable moderadora donde el efecto de la variable predictora $X$ sobre la variable respuesta $Y$ resulta significativo.

La interpretación del efecto interactivo se realiza sobre un gráfico (figura 4), mostrando la relación entre la variable predictora y la variable dependiente para ciertos niveles seleccionados de la variable moderadora (Aiken y West, 1991; Jaccard y Turrisi, 2003). Si las rectas son significativamente diferentes entre sí, esto se entiende como el término de la interacción significativa (Aiken y West, 1991; Dawson, 2014). 
La figura 4 muestra las pendientes que permiten interpretar la interacción entre la variable predictora RELAGEN y la moderadora EXPREVI, en su relación con la variable dependiente DESEMP. En dicha figura se puede observar el comportamiento del efecto que produce el factor independiente (RELAGEN) en la variable dependiente DESEMP, dados diferentes niveles de experiencia previa de los socios promotores de la cooperativa.

Dado un menor nivel de experiencia previa adquirida por los emprendedores antes de crear la cooperativa, existe en ellos una mayor preocupación o interés por establecer y forjar relaciones con diferentes agentes del entorno, con una pendiente claramente positiva, que indica cómo los emprendedores más inexpertos o con menor experiencia conceden mayor importancia a la relación con dichos agentes externos, y así el impacto en los resultados o la performance de la empresa resulta evidente, mientras que para los emprendedores más expertos y con mayor experiencia laboral, técnico-profesional o de gestión empresarial, el interés por este tipo de relaciones se atenúa, o incluso se reduce con el tiempo, dada la pendiente negativa, produciéndose un efecto más atenuado en la performance de la empresa.

\section{Figura 4. Prueba de pendientes simple (2-vías). Variable dependiente: DESEMP}

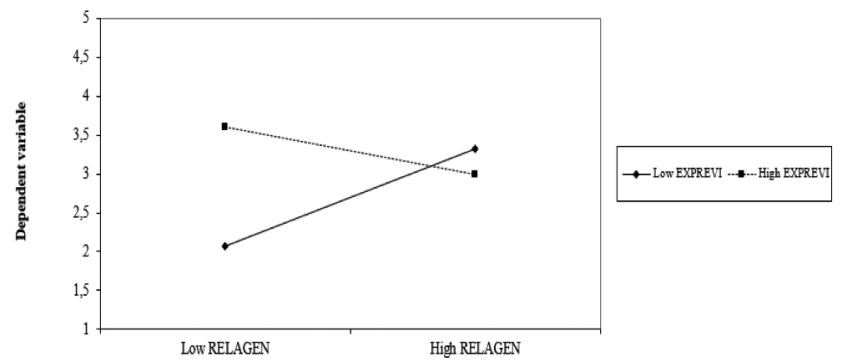

FUENTE: Elaboración propia, a partir de Dawson (2014). 


\section{Conclusiones}

La creación de una CTA no responde exactamente a los cánones del emprendimiento en empresas tradicionales, lo que, a la hora de su estudio, esto dificulta el posible encaje en los modelos generales sobre emprendimiento o entrepreneurship. Por esa razón, y en la práctica, el estudio de las cooperativas de trabajo asociado se ha visto velado por el programa general de investigación en organización de empresas y el emprendimiento, que se fundamenta en la empresa capitalista tradicional, sin tener en cuenta que la CTA y su proceso de emprendimiento puede considerarse un "nuevo fenómeno" de dicho programa, dificultando el desarrollo de un marco conceptual robusto que sirva de motor a la investigación del ámbito del emprendimiento cooperativo de trabajo asociado, y revele el potencial que encierra este tipo de empresa como forma organizativa12.

Contando con ese condicionante, este trabajo realiza una aportación al conocimiento sobre el comportamiento empresarial, plasmado en el proceso de emprendimiento de la CTA, y su relación con el rendimiento de las empresas y las expectativas de los emprendedores cooperativos, para quienes los incentivos tienen un eminente carácter cualitativo, siendo el propósito principal de su actividad emprendedora conseguir una forma de subsistencia y desarrollo personal, laboral y profesional, desde una óptica participativa.

Explorar tales relaciones entre el comportamiento empresarial y el rendimiento resulta oportuno, como señalan Lumpkin y Dess (1996), dadas las condiciones competitivas que enfrentan las empresas de todos los tamaños en la economía actual.

El objetivo de este trabajo ha sido construir sobre teorías e investigaciones previas para, en primer lugar, aclarar la naturaleza multidimensional del proceso de emprendimiento en las CTA, y en segundo lugar sugerir modelos de contingencia alternativos que, en la línea de otros trabajos de orientación emprendedora proporcionen información adicional sobre la relación orientación emprendedora - rendimiento.

Es por ello que, y desde una visión multidimensional, se propone un modelo singular que analiza dicho comportamiento empresarial, u orientación emprendedora, mediante un conjunto de factores seleccionados de las teorías más relevantes dentro del enfoque sociocultural e institucional, estando éstos presentes en el proceso de emprendimiento, en las fases de gestación, creación y puesta en marcha de la CTA.

12.- Aun contando con aportaciones tan interesantes como las de Guzmán, Santos y Barroso (2016); Orellana y Monreal (2017); y Bretos et al. (2018), entre otros. 
Los esfuerzos de investigación dirigidos a comprender la dimensionalidad del proceso de emprendimiento en las cooperativas de trabajo asociado contribuirán a un mayor desarrollo teórico en el campo del emprendimiento. Según (Lumpkin y Dess (1996) "la investigación para diseñar escalas de medida, explorar los procesos subyacentes asociados con la actividad empresarial, y fundamentalmente reconocer la naturaleza multidimensional del comportamiento empresarial también mejorará nuestra comprensión de la orientación emprendedora y su relación con el desempeño organizacional".

Con independencia de los resultados obtenidos, este trabajo ha supuesto un esfuerzo en esa dirección.

\section{Bibliografía}

AIKEN, L.S. \& WEST, S.G. (1991): Multiple regression: Testing and interpreting interactions, Newbury Park. London, Sage.

ALCHIAN, A. \& DEMSETZ, H. (1972): "Production, information cost and economic organization", Quarterly Journal of Economics, 63 (5), 777-795.

ALONSO, M.J. y GALVE, C. (2008): "El emprendedor y la empresa: una revisión teórica de los determinantes de su constitución", Acciones e Investigaciones Sociales, 26, julio, 5-44.

ARMIGNTON, C. \& ACS, Z.J. (2002): "The determinants of regional variation in New Firm Formation", Regional Studies, 36 (1), 33-45.

ARZUBIAGA, U., ITURRALDE, T. y MASEDA, A. (2012): "La medición de la Orientación emprendedora en las empresas familiars", Revista de Empresa Familiar, 2 (2), 57-31.

BATHES, T. (1997): "Financing small business creation: The case of Chinese and Korean immigrant entrepreneurs", Journal of Business Venturing, 109-124.

BAUER, D.J. \& CURRAN, P.J. (2005): "Probing interactions in fixed and multilevel regression: Inferential and graphical techniques", Multivariate Behavioral Research, 40, 373-400.

BIRLEY, S. (1985): "The role of Networks in the entrepreneurial process", Journal of Business Venturing, 1, 107-117.

BOSMA, N., VAN STEL, A. \& SUDDLE, K. (2008): "The geography of new firm formation: Evidence from independent start-ups and new subsidiaries in the Netherlands", International Entrepreneurship Management Journal, 4, 129-146. 
BRETOS, I., DIAZ-FONCEA, M., MARCUELLO, CH. \& MARCUELLO, C. (2018): "Cooperativas, capital social y emprendimiento: una perspectiva teórica", REVESCO, Revista de Estudios Cooperativos, 128, 76-98. DOI: 10.5209/REVE.59775.

BRUNET, I. \& ALARCON, A. (2004): "Teorías sobre la figura del emprendedor", Papers, 73, 81-103.

CHEN,M-H., CHANG,Y-Y. \& LIN, Y-C.(2018): "Exploring creative entrepreneurs' happiness: cognitive style, guanxi and creativity", International Entrepreneurship and Management Journal, 14(4), 1089-1110. DOl: https://doi.org/10.1007/s11365-017-0490-3.

CHIN, W.W. (1998): "The partial least squares approach to structural equation modeling". In Marcoulides, G.A. (Ed.), Modern methods for business research. Mahwah, Lawrence Erlbaum Associates. NJ, 295-336.

COHEN, J. (1998): Statistical power analysis for the behavioral sciences. Hilsdale, NJ: Lawrence Erlbaum Associates, $2^{\mathrm{a}}$ edition.

COHEN, J., COHEN, P., WEST, S.G. \& AIKEN, L.S. (2003): Applied multiple regression correlation analysis for the behavioral sciences, $3^{\mathrm{a}} \mathrm{Ed}$. Mahwah: Lawrence Erlbaum.

COLLINS, O.F., MOORE, D.G. \& UNWALLA, D.B. (1964): The Enterprising Man, East Lansing, M.I., Michigan State University Business Studies.

COOPER, A.C. (1985): "The Role of incubator organizations in the founding of growth oriented firms". Journal of Business Venturing, 1 (1), 75-86.

COOPER, A.C. \& DUNKELBERG, W.C. (1987): "Entrepreneurial research: old questions, new answers and methodological issues", Journal of Small Business, 1987, 11 (3), 11-23.

COVIN, J.G. \& SLEVIN, D.P. (1989): "Strategic management of small firms in hostile and benign environments", Strategic Management Journal, 10 (75), 75-87.

COVIN, J.G. \& WALES, W.J. (2012): "The measurement of entrepreneurial orientation", Entrepreneurship Theory and Practice, 36 (4), 677-702.

DAWSON, J.F. (2014): "Moderation in management research: What, why, when and how", Journal of Business and Psychology, 29, 1-19.

EAGLY, A.H. \& CARLI, L.L. (2003): "The female leadership advantage: An evaluation of the evidence", Leadership Quarterly, 14 (6), 807-834.

EVANS, D. \& LEIGHTON, H. (1989): "Some Empirical Aspects of Entrepreneurship", American of Economic Review, 79 (3), 519-535.

FAMA, E. \& JENSEN, M. (1983): "Agency Problems and Residual Claims", Journal of Law and Economics, 26, 327-349.

FERNÁNDEZ-MESA, A., ALEGRE, J. y CHIVA, R. (2012): "Orientación emprendedora, capacidad de aprendizaje organizativo y desempeño innovador", Journal of Technology Management \& Innovation, (2), 157-170. 
FORNELL, C. (1982): "A Second Generation of Multivariate Analysis: An Overview", en C. Fornell, C. (eds.): A Second Generation of Multivariate Analysis, 1, 1-21. New York: Praeger Publishers.

GARTNER, W.B. (1985): "A conceptual framework for describing the phenomenon of new venture creation", Academy of Management Review, 10 (4), 696-706.

GEORGE, G., WOOD, D.R. \& KHAN, R. (2001): "Networking strategy of boards: Implications for small and medium-sized enterprises", Entrepreneurship \& Regional Development, 13 (3), 269-285.

GREEN, K.M., COVIN, J.G. \& SLEVIN, D.P. (2008): "Exploring the relationship between strategic reactiveness and entrepreneurial orientation: The role of structure-style fit", Journal of Business Venturing, 23 (3), 356-383.

GUZMAN, C., SANTOS, F.J. y BARROSO, M. (2016): “Cooperativismo, factor empresarial y Desarrollo económico: propuesta de un modelo teórico de enlace", REVESCO, Revista de Estudios Cooperativos, 122, 110-134. DOI: 10.5209/rev_REVE_2016.v122.52018.

HAIR, J.F., HULT, G., RINGLE, C.M. \& SARSTEDT, M. (2014): A Primer on Partial Least Squares Structural Equation Modeling (PLS-SEM), Thousand Oaks: CA: Sage.

HAIR, J.F., HULT, G., RINGLE, C.M., SARSTEDT, M., CASTILLO, J., CEPEDA, G. \& ROLDAN, J.L. (2019): Manual de Partial Least Squares Structural Equation Modeling (PLS-SEM), $2^{\mathrm{a}}$ edición, SAGE Publishing, DOI: 10.3926/oss.37.

HART, S.L. (1992): "An integrative framework for strategy-making processes", The Academy of Management Review, 17 (2), 327-351.

HAYES, A.F. (2009): "Beyond Baron and Kenny: Statistical Mediation Analysis in the New Millennium", Communication Monographs, 76 (4), 408-420.

HAYTON, J.C., GEORGE, G. \& ZAHRA, S.A. (2002): "National culture and entrepreneurship: A review of behavioral research", Entrepreneurship, Theory and Practice, 26 (4), 33-52.

HERNANDEZ PERLINES, F. (2014): "Orientación emprendedora de las cooperativas agroalimentarias con actividad exportadora", CIRIEC-España, Revista de Economía Pública, Social y Cooperativa, 80, 29-55.

JACCARD, J. \& TURRISI, R. (2003): Interaction effects in multiple regression, $2^{\mathrm{a}}$ ed: Thousand Oaks, CA: Sage.

JENSEN, M. \& MECKLING, W. (1976): "Theory of the Firm: Managerial Behaviour, Agency Costs, and Ownership Structure", The Journal of Financial Economics, vol. 3. [Versión en castellano y extractado en Putterman, L. (ed.) (1994): La Naturaleza Económica de la Empresa. Alianza Editorial. Madrid.

JOHNSON P.O. \& NEYMAN J. (1936):" Tests of certain linear hypotheses and their applications to some educational problems", Statistical Research Memoirs, 1, 57-93. 
LAWRENCE, P.R. \& LORSCH, J.W. (1967): Organization and environment: Managing differentiation and integration, Boston, Harvard Graduate School [Versión en castellano (1976): Organización y ambiente. Labor, Barcelona].

LUMPKIN, G.T. \& DESS, G.G. (1996): "Clarifying the entrepreneurial orientation construct and linking it to performance", Academy of Management Review, 21 (1), 135-172.

LUMPKIN, G.T. \& DESS, G.G. (2001): "Linking two dimensions of entrepreneurial orientation to firm performance: The moderating role of environment and industry life cycle", Journal of Business Venturing, 16 (5), 429-451.

MARCH, J.G. \& SIMON, H.A (1958): Organizations, New York: John Wiley and Sons. 1958 [Versión en castellano (1977): Teoría de la Organización. Ariel. Barcelona].

MARCH, I. y YAGÜE, R. (2009): "Desempeño en empresas de economía social. Un modelo para su medición", CIRIEC-España, Revista de Economía Pública, Social y Cooperativa, 64, 105-131.

MASON, C.H. \& PERREAULT, J.W.D. (1991): "Collinearity, Power, and Interpretation of Multiple Regression Analysis", Journal of Marketing Research, 28 (3), 268-280.

MLLLER, D. (1983): "The correlates of entrepreneurship in three types of firms", Management Science, 29 (7), 770-791.

MINNITI, M., (2005): "Entrepreneurship and network externalities", Journal of Economic Behavior and Organization. 57 (1), 1-27.

NORTH, D.C. (1990): Institutions, Institutional Change and Economic Performance, Cambridge University Press, Cambridge (UK).

NUENO, P. (1996): "Evolución de los conceptos de management", Management Review, 1, eneroabril, 73-83.

O'SHEA, D.J., BUCKLEY, F. \& HALBESLEBEN, J. (2017): "Self-regulation in entrepreneurs: Integrating action, cognition, motivation, and emotions", Organizational Psychology Review, 250-278. DOI: https://doi.org/10.1177/2041386617705434.

OCDE (1999): Estimular el espíritu empresarial, OCDE, París.

ORELLANA, W.E. (2002): "El control de empresas de trabajo asociado. Revisión crítica desde una perspectiva de agencia, de los principales mecanismos de control, y análisis de sus repercusiones organizativas", Revista de Economía Pública Social y Cooperativa, 42, 49-80.

ORELLANA, W.E. y MONREAL, M. (2007): Control, incentivos y comportamiento en la empresa, ACDE, Valencia.

ORELLANA, W.E. y MARTÍNEZ DE LEJARZA, J. (2013): "Teorías de entrepreneurship y cooperativismo de trabajo asociado. Fundamentos teóricos y evidencias empíricas en la creación de CTA", CIRIEC-España, Revista de Economía Pública, Social y Cooperativa, 78, 11-36. 
ORELLANA, W.E. y MONREAL, M. (2017): Trabajo asociado y emprendimiento cooperativo en la Comunidad Valenciana. Análisis de la cooperativa de trabajadores como fórmula empresarial y análisis de sus procesos de dirección y emprendimiento, CIRIEC-España, Valencia.

OUCHI, W. (1980): "Markets, bureaucracies and clans", Administrative Science Quarterly, 25, 121 141.

POTHOFF, R.F. (1964): "On the Johnson-Neyman technique and some extensions thereof", Psychometrika, 29, 241-256.

PREACHER, K.J. \& HAYES, A.F. (2008): "SPSS and SAS procedures for estimating indirect effects in simple mediation models", Behavior Research Methods, Instruments, and Computers, 36, 717731.

RAUCH, A., WIKLUND, J., LUMPKIN, G.T. \& FRESE, M. (2009): "Entrepreneurial orientation and business performance: An assessment of past research and suggestions for the future", Entrepreneurship Theory \& Practice, 33, 761-787.

REYNOLDS, P., MILLER, B. \& MAKI, W.R. (1995): "Explaining regional variation in business births and deaths: US 1976-88", Small Business Economics, 7, 389-407.

RIPOLLES, M. y BLESA, A. (2006): "Redes personales del empresario y orientación emprendedora en las nuevas empresas", Cuadernos de Economía y Dirección de la Empresa, 26, 73-94.

RUMELT, R.P. (1991): "How much does industry matter? Strategic Management Journal, 12, 167-185.

SHEPHERD, D.A. \& PATZELT, H. (2018) "Motivation and Entrepreneurial Cognition". En: Entrepreneurial Cognition. Exploring the Mindset of Entrepreneurs, Palgrave Macmillan, Cham. 51-103. DOI: https://doi.org/10.1007/978-3-319-71782-1_3.

SCHMALENSEE, R. (1985): "Do markets differ much?", American Economic Review, 75 (3), 341-351.

SCHUMPETER, J. (1934): The Theory of Economic Development. Cambride: Harvard University Press. (New York: Oxford University Press, 1961) First published in German, 1912.

SHAPERO, A. (1975): "The displaced, uncomfortable entrepreneur", Psychology Today, 9 (6), 8388.

SHAPERO, A. \& SOKOL, L. (1982): "The social dimensions of entrepreneurship". In Kent, Sexton y Vesper (eds.), The Encyclopedia of Entrepreneurship. Englewood Cliffs: Prentice Hall, 72-90.

SIMON, H.A. (1947): Administrative Behaviour. A study of decision making processes in administrative organization, New York: Harper \& Brothers. [Versión en castellano (1972): El comportamiento administrativo. Estudio de los procesos de adopción de decisiones en la organización administrativa. Aguilar. Madrid].

SMART, D.T. \& CONANT, J.S. (1994): "Entrepreneurial orientation, distinctive marketing competencies and organizational performance", Journal of Applied Business Research, 10, 28-38. 
STAM, W. \& ELFRING, T. (2008): "Entrepreneurial orientation and new venture performance: the moderating role of intra- and extraindustry social capital", Academy of Management Journal, 51 (1), 97 111.

TERVO, H. (2006): "Regional unemployment, self-employment and family background", Applied Economics, 38 (9), 1055-1062.

VECCHIO, R.P. (2003): "In search of gender advantage", The Leadership Quarterly, 14, 835-850.

VECIANA, J.M. (1996): "Emprendedor o empresario", Innovando, Boletín del Centro de Desarrollo del Espíritu Empresarial de la Universidad de ICESI, nº 17.

VERHEUL, L., WENNEKERS, S., AUDRETSCH, D.B. \& THURIK, R. (2002): An Eclectic Theory of Entrepreneurship: Policies, Institutions and Culture, University of Illinois at Urbana-Champaign's Academy for Entrepreneurial Leadership Historical Research Reference in Entrepreneurship.

VERHEUL, I. (2005): "Is there a (fe)male approach?", Understanding gender differences in entrepreneurship, ERIM Ph.D. Series Research in Management, Erasmus University Rotterdam, The Netherlands.

WALES, W., GUPTA, V. \& MOUSA, F.T. (2013): "Empirical research on entrepreneurial orientation: An assessment and suggestions for future research", International Small Business Journal, 31 (4), 357-383.

WANG, C. (2008): "Entrepreneurial orientation, learning orientation, and firm performance", Entrepreneurship Theory and Practice, 32 (4), 635-657.

WESTHEAD, P. \& WRIGHT, M. (1998): "Novice, portfolio, and serial founders: are they different?", Journal of Business Venturing, 13 (3), 173-204.

WILLIAMSON, O.E. (1985): The Economic Institutions of Capitalism, Nueva York, The Free Press, [Traducción al castellano (1989): Las instituciones económicas del capitalismo. Fondo de Cultura Económica, México]. 
